\title{
Patient-specific Alzheimer-like pathology in trisomy 21 cerebral organoids reveals BACE2 as a gene dose-sensitive AD suppressor in human brain
}

\author{
Ivan Alić $\mathbb{D}^{1,2,3}$ - Pollyanna A. Goh ${ }^{2,4}$. Aoife Murray $\mathbb{B}^{1} \cdot$ Erik Portelius $^{5} \cdot$ Eleni Gkanatsiou ${ }^{5}$ Gillian Gough $\mathbb{1}^{1}$. \\ Kin Y. Mok $\mathbb{1}^{4,6} \cdot$ David Koschut $^{1} \cdot$ Reinhard Brunmeir ${ }^{1} \cdot$ Yee Jie Yeap ${ }^{1} \cdot$ Niamh L. O'Brien $^{2,4} \cdot$ Jürgen Groet $^{2,4}$.

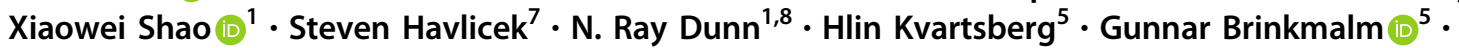 \\ Rosalyn Hithersay ${ }^{4,9,10}$. Carla Startin $\mathbb{1}^{4,9} \cdot$ Sarah Hamburg ${ }^{4,9} \cdot$ Margaret Phillips $^{11} \cdot$ Konstantin Pervushin ${ }^{11}$.

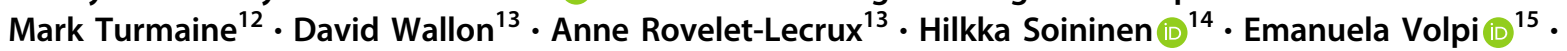 \\ Joanne E. Martin $\mathbb{1}^{2} \cdot$ Jia Nee Foo ${ }^{1,7} \cdot$ David L. Becker $^{1} \cdot$ Agueda Rostagno $^{16} \cdot$ Jorge Ghiso $^{16}{ }^{16}$ Željka Krsnik ${ }^{17}$. \\ Goran Šimić $^{17}$ • Ivica Kostović ${ }^{17}$ • Dinko Mitrečić $\mathbb{C}^{17}{ }^{17}$. LonDownS Consortium ${ }^{4}$ • Paul T. Francis ${ }^{18}$. \\ Kaj Blennow $\mathbb{B}^{5} \cdot$ Andre Strydom $\mathbb{C}^{4,9,10} \cdot$ John Hardy ${ }^{4,6} \cdot$ Henrik Zetterberg ${ }^{5,6} \cdot$ Dean Nižetić $\mathbb{B}^{1,2,4}$
}

Received: 29 January 2020 / Revised: 18 May 2020 / Accepted: 29 May 2020 / Published online: 10 July 2020

(c) The Author(s) 2020. This article is published with open access, corrected publication 2021

\begin{abstract}
A population of more than six million people worldwide at high risk of Alzheimer's disease (AD) are those with Down Syndrome (DS, caused by trisomy 21 (T21)), 70\% of whom develop dementia during lifetime, caused by an extra copy of $\beta$ amyloid-(A $\beta)$-precursor-protein gene. We report AD-like pathology in cerebral organoids grown in vitro from noninvasively sampled strands of hair from $71 \%$ of DS donors. The pathology consisted of extracellular diffuse and fibrillar A $\beta$ deposits, hyperphosphorylated/pathologically conformed Tau, and premature neuronal loss. Presence/absence of AD-like pathology was donor-specific (reproducible between individual organoids/iPSC lines/experiments). Pathology could be triggered in pathology-negative T21 organoids by CRISPR/Cas9-mediated elimination of the third copy of chromosome 21 gene $B A C E 2$, but prevented by combined chemical $\beta$ and $\gamma$-secretase inhibition. We found that T21 organoids secrete increased proportions of $A \beta$-preventing (A $\beta 1-19)$ and $A \beta$-degradation products $(A \beta 1-20$ and $A \beta 1-34)$. We show these profiles mirror in cerebrospinal fluid of people with DS. We demonstrate that this protective mechanism is mediated by $B A C E 2$-trisomy and cross-inhibited by clinically trialled BACE1 inhibitors. Combined, our data prove the physiological role of $B A C E 2$ as a dose-sensitive AD-suppressor gene, potentially explaining the dementia delay in $~ 30 \%$ of people with DS. We also show that DS cerebral organoids could be explored as pre-morbid AD-risk population detector and a system for hypothesis-free drug screens as well as identification of natural suppressor genes for neurodegenerative diseases.
\end{abstract}

These authors contributed equally (ordered alphabetically): Aoife Murray, Erik Portelius, Ivan Alić, Pollyanna A. Goh

These authors jointly supervised this work: John Hardy, Henrik Zetterberg, Dean Nižetić

A full list of LonDownS Consortium members and their affiliations appear below the acknowledgements section.

Supplementary information The online version of this article (https:// doi.org/10.1038/s41380-020-0806-5) contains supplementary material, which is available to authorized users.

Dean Nižetić

d.nizetic@qmul.ac.uk

Extended author information available on the last page of the article

\section{Introduction}

Production [1-3], and degradation [4] of $\beta$-amyloid peptides $(\mathrm{A} \beta)$ are among the central processes in the pathogenesis of Alzheimer's disease (AD). The canonical A $\beta$ peptide is produced after sequential cleavage of the $\beta$-amyloid precursor-protein (APP) by $\beta$-secretase and $\gamma$-secretase, generating a peptide that most often begins 99 amino acids (aa) from the C-terminus of APP with Asp1 and contains the next 37-42 aa of the APP sequence, generating a range of peptides (A $\beta 1-37,38,39,40$, and 42). The longer of these peptides can be detected in toxic amyloid aggregates in the brain, associated with $\mathrm{AD}$ and other neurodegenerative disorders [5]. As APP gene is located on human 

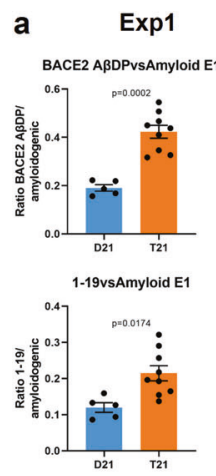

ACE2vsAmyloid E

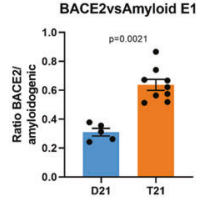

pha vsAmyloid E1
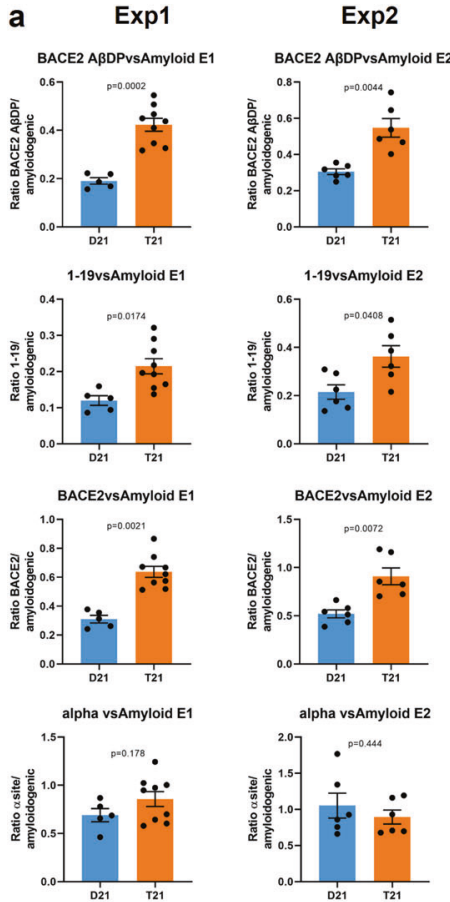

1-19vsAmyloid E2

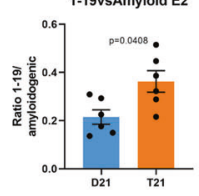

BACE2vsAmyloid E2

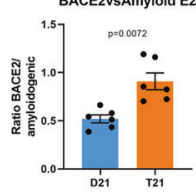

alpha vsAmyloid E2
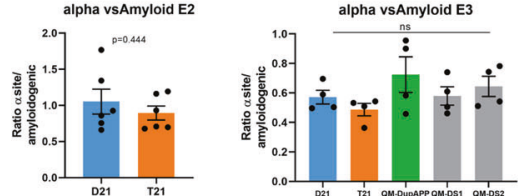

Fig. $1 \mathrm{~A} \beta$ peptide profiles secreted by trisomy 21 cerebral organoids. a Using A $\beta$ IP-MS spectra from organoid (see Supplementary Figs. 1, 2, 4) conditioned media (CM), ratios were calculated of areas under the peak between the non-amyloidogenic and amyloidogenic peptides within a single mass-spectrogram. IP-MS spectra were produced for three timepoints (four timepoints in exp3) for each iPSCderived organoid line, in each of three independent experiments (each experiment starting at the point of undifferentiated iPSC). The team performing the IP-MS analysis was blinded to the genotypes in all experiments. BACE2-A $\beta D P$ (clearance) products $=\left[\begin{array}{lll}1-20 & \& & 1-34\end{array}\right]$, total $\mathrm{BACE} 2=[1-19 \& 1-20 \& 1-34]$, amyloidogenic peptides $=$ [1-38 \& 1-39 \& 1-40 \& 1-42], $\alpha$-site products $=[1-16 \& 1-17]$. Exp1 and $\operatorname{Exp} 2 p$ values: Holm-Bonferroni sequential corrections $(\alpha=0.05)$ of two-tailed student $t$ test comparisons. Exp3: Holm-corrected $p$ values after one-way ANOVA. Error bars: standard error. Combined data for

chromosome 21, people with Down Syndrome (DS, caused by trisomy 21 (T21)) are born with one extra copy of this gene, which increases their risk of developing AD. Non-DS (euploid) people inheriting triplication of the APP gene alone (DupAPP) develop AD symptoms by age 60 with $100 \%$ penetrance. Paradoxically, only $\sim 70 \%$ of people with DS develop clinical dementia by age 60 , suggesting the presence of other unknown chromosome 21-located genes that modulate the age of dementia onset [6,7]. A number of secretases participate in the physiological cleavage of APP $[1,8]$, generating various peptides involved in neuronal pathology. BACE1 is the main $\beta$-secretase in the brain [9], whereas the expression and function of its homologue BACE2 (encoded by a chromosome 21 gene) remain less clear $[10,11]$. At least three different activities of BACE2 were recorded with regards to APP processing: as an auxiliary $\beta$-secretase (pro-amyloidogenic), as a $\theta$-secretase (degrading the $\beta$-CTF and preventing the formation of

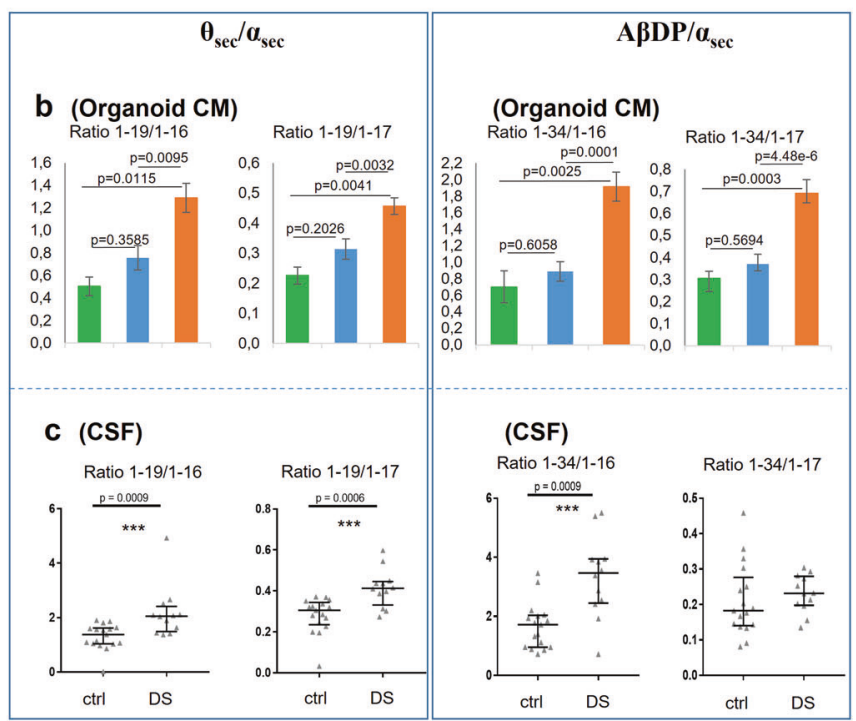

-D21

T21

QM-DUPAPP

non-isogenic DS

the isogenic iPSC lines for all three experiments passed the Holm-Bonferroni correction ( $\alpha=0.05$ ) of sequential two-tailed student $t$ test comparisons of each peptide ratio shown in Fig. 1a (available on request). T21 and D21: isogenic iPCS derived from a single mosaic individual with DS published previously (Murray A et al. 2015), QMDS1 and QM-DS2: unrelated DS iPSC, DupAPP: FEOAD iPSC. b All three experiments in Fig. 1a were combined to calculate the ratios of BACE2-related non-amyloidogenic peptides (1-19 or 1-34) to BACE2-unrelated non-amyloidogenic peptides (1-16 or 1-17) in organoid CM. Holm-corrected $p$ values after one-way ANOVA are shown. Error bars: standard error. c Same ratios as in part ' $a$ ' were calculated on IP-MS spectra obtained from cerebrospinal fluid samples of people with DS $(n=17)$ and age-matched normal controls $(n=12)$. Data are presented as mean $\pm 1 \mathrm{SD}$.

$\mathrm{A} \beta$ ), and as $\mathrm{A} \beta$-degrading protease (A $\mathrm{DPP}$ ) (degrading synthetic $\mathrm{A} \beta$-peptides at extremely acidic $\mathrm{pH}$ ). It remains unclear which of these activities reflect the role of BACE2 in AD. The potential activity of BACE2 as an antiamyloidogenic $\theta$-secretase can be predicted from studies on a variety of transfected cell lines that overexpress $A P P$, and artificially manipulate the dose of BACE2 [12-15]. These studies uncovered that BACE2 can cleave the product of $\beta$ secretase (APP $\beta$-CTF) between aa19 and aa20, generating a 1-19 fragment [13-15], thereby potentially preventing the formation of amyloidogenic $\mathrm{A} \beta$, and degrading the $\beta$-CTF that has been implicated in neuronal toxicity, and impairment of several neuronal functions, such as axonal transport and autophagy [16]. When offered synthetic A $\beta 40 / 42$ peptides in solution, purified BACE2 protein can rapidly degrade them by cutting after aa20 and aa34, to generate the 1-20 and 1-34 peptide products, but only at very acidic $\mathrm{pH}$ (3.5-4). In this reaction, BACE2 is 150 -fold more efficient 
than BACE1, which is also capable of this cleavage, upon conditions of increased enzyme concentration/time [12, 14]. Neither of these two putative anti-amyloidogenic actions of BACE2 (the $\theta$-secretase activity, generating aa1-19, or the $\mathrm{A} \beta$-degrading protease activity ( $\mathrm{A} \beta \mathrm{DP}$ or $\mathrm{A} \beta$ clearance) generating aa1-20 and aa1-34), have yet been demonstrated to be the functional role of BACE2 under physiologically fluctuating gene doses in vivo in the human brain. A naturally occurring form of gene overdose for both $A P P$ and $B A C E 2$ is DS, caused by the trisomy of human chromosome 21 (T21) that harbours both $A P P$ and BACE2 genes. As increased levels of soluble $A \beta$ were observed already in foetal brains in DS [17], we examined cerebral organoids grown from induced pluripotent stem cells (iPSC) generated by non-integrational reprogramming of primary cells donated by people with DS, including an isogenic DS (T21) iPSC model [18], as a platform to analyse the T21specific effects on APP proteolytic processing.

\section{Results}

\section{Trisomy 21 (but not DupAPP) skews the ratios of $A \beta$ non-amyloidogenic peptides}

We compared organoids from isogenic iPSC clones, derived from the same individual with DS, mosaic for T21 and normal disomy 21 (D21) cells [18]. Cerebral organoids were derived following a published protocol [19], and shown to contain neurons expressing markers of all six layers of the human cortex (Supplementary Fig. 1) and no significant difference in the proportions of neurons and astrocytes between the D21 and T21 organoids (Supplementary Fig. 2). The integrity and copy number of the iPSC lines were validated at the point of starting the organoid differentiation, for chromosome 21 (Supplementary Fig. 3), and the whole genome (available on request). T21/ D21 status was further verified by interphase Fluorescence In Situ Hybridization (FISH) on mature organoid slices, (Supplementary Fig. 4a). The C-terminal region of APP can be processed by the sequential action of different proteases to produce a range of protein fragments and peptide species, including $A \beta$ (Supplementary Fig. 5). A $\beta$ peptide profiles were analysed from organoid-conditioned media (CM) whereby each CM sample was taken from a 6 $\mathrm{cm}$ dish culturing a pool of 12-16 organoids derived from one iPSC clone, in total: $n=15 \mathrm{CM}$ samples for Exp1 (three trisomic isogenic clones, two disomic isogenic clones, three timepoints each), $n=12 \mathrm{CM}$ samples for Exp2 (two trisomic isogenic clones, two disomic isogenic clones, three timepoints each) and $n=20 \mathrm{CM}$ samples for Exp3 (one trisomic isogenic clone, one disomic isogenic clone, one DupAPP clone, one line each for two different unrelated DS individuals, four timepoints each). CM was collected at timepoints between days 100-137 of culturing and analysed using immunoprecipitation in combination with mass spectrometry (IP-MS) [20]. Please see "Methods" and "Supplementary Data" sections for more detailed explanations, and statistical controls used for individual iPSC line-to-line comparisons (Fig. 1a). Relative ratios were calculated of areas under the peak between the peptides of interest within a single mass spectrum (raw data example in Supplementary Fig. 6d), therefore unaffected by the variability in the total cell mass between wells growing organoids. The proportions of non-amyloidogenic peptides with the signature of BACE2 cleavage products, both as a putative $\theta$-secretase (as reflected by the $A \beta 1-19$ product) and putative $\mathrm{A} \beta \mathrm{DP}$ or $\mathrm{A} \beta$-clearance products $(\mathrm{A} \beta 1-20$ \& 1-34), or combined, (relative to the sum of $A \beta$ amyloidogenic peptides (A $\beta 1-38 \& 1-39 \& 1-40 \& 1-42))$ were approximately doubled in CM from T21 organoids, compared with isogenic normal controls, and reached levels of $>80 \%$ of the amyloidogenic peptide levels (Fig. 1a). This result was fully reproduced in three independent experiments, each starting from undifferentiated iPSCs (three vertical columns of graphs in Fig. 1a). In experiment 3, more recently generated iPSC lines from different individuals were introduced; from a euploid patient with FEOAD caused by DupAPP [21], and from two unrelated people with DS (Supplementary Figs. 1-3). The 1-34 \& 1-20/ amyloidogenic ratios were not significantly different between D21 and DupAPP lines, suggesting the third copy of the $A P P$ gene alone did not cause any change in this ratio. Ratios of 1-34 \& 1-20/amyloidogenic peptides and combined BACE2-products/amyloidogenics were significantly increased in T21 clones (combining all three T21 individuals) compared with D21 or DupAPP lines (Fig. 1a). The ratio of 1-19/amyloidogenics was significantly higher in T21 clones from the isogenic model, compared with its disomic isogenic control, and compared with DupAPP, but it was unchanged in the other two unrelated DS iPSC lines (see also Supplementary Information for a more-detailed explanation). As the proportions of BACE2-unrelated $\alpha$-site cleavage products $(1-16,1-17)$ were not different between T21 and isogenic D21 organoids (in any of the three experiments) (Fig. 1a), it can be predicted that the increased presence of 1-19, 1-20, and 1-34 peptides in T21 contributes towards an overall increase in soluble peptides that are nonamyloidogenic. The validity of this prediction was tested by an independent biochemical method (ELISA), by measuring the $\mathrm{A} \beta$-peptide concentrations within the isogenic T21:D21 organoid $\mathrm{CM}$ comparison, which showed an increase in absolute concentrations caused by $\mathrm{T} 21$ for each $\mathrm{A} \beta \mathrm{1}-38$, $1-40$, and $1-42$, with no difference in the $A \beta 1-42 / 1-40$ ratio between T21 and isogenic D21 clones, mirroring the readout in the absolute levels of IP-MS peaks (Supplementary Fig. 6). 

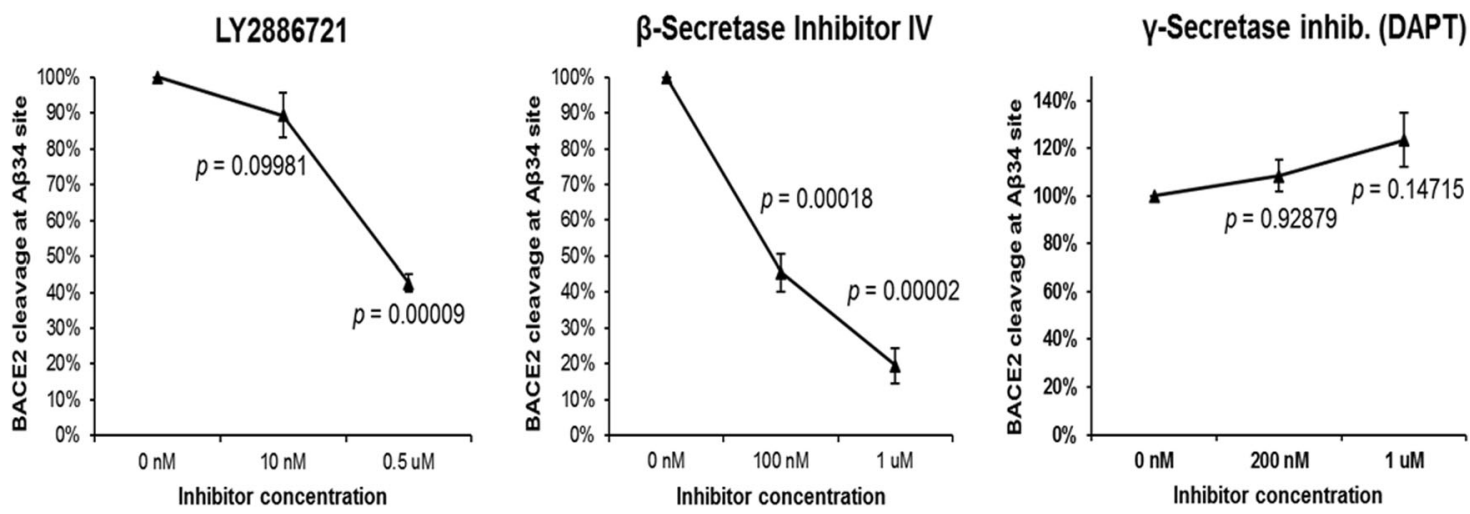

Fig. 2 FRET-based assay for BACE2 cleavage. A newly custom designed FRET reagent (spanning the A $\beta 34$ site) was digested at $\mathrm{pH}=3.5$ by the human BACE2 in presence or absence of the stated inhibitors for $2 \mathrm{~h}$. Enzyme activity was defined by measuring the fluorescence increase before and after the incubation. Blank-subtracted

Analysis of IP-MS area under peak (used in Fig. 1 to calculate relative ratios) showed a near linear correlation when plotted against absolute peptide concentrations measured by ELISA, for each $A \beta 1-38,1-40$, and 1-42 (Supplementary Fig. 6), validating our relative ratio calculations by an independent biochemical method.

To estimate the contribution of BACE2 towards the antiamyloidogenic pathway relative to other anti-amyloidogenic cleavages at the $\alpha$-site, we calculated the peptide ratios of $1-19 / 1-16$ or $1-17$ ( $\theta$ secretase/ $\alpha$ secretase products) and $1-34 / 1-16$ or $1-17$ (BACE2-A $\beta D P / \alpha$ secretase products). We observed that $\mathrm{T} 21$ organoids produce statistically highly significant increases in all four of these ratios, relative to isogenic D21, or non-isogenic DupAPP organoids (Fig. 1b). Therefore, we conclude that T21 causes these effects in our organoid system. The D21 ratios were not significantly different to DupAPP, suggesting that the third copy of genes other than $A P P$ causes these effects. These peptide profiling data strongly favour the hypothesis of a genetic dosesensitive anti-amyloidogenic action of BACE2.

\section{Non-amyloidogenic $A \beta$ peptide ratios mirror between T21 organoids and DS-CSF}

In order to assess if the peptide ratio differences from Fig. $1 \mathrm{~b}$ have any relevance in vivo, we analysed the $A \beta$ peptide profiles immunoprecipitated from human cerebrospinal fluid (CSF). We have previously produced IP-MS data on CSF from people with DS and age-matched controls [22]. We repeated the calculations shown for organoids in Fig. 1b, on IP-MS results from CSF samples from DS $(n=$ 17) and age-matched euploid people $(n=12)$. All four relative ratio calculations showed an increase in peptide ratios in CSF from people with DS, compared with agematched euploid controls, of which three comparisons were statistically highly significant (Fig. 1c). This suggests that in fluorescence units were normalized to the control digest and a one-way ANOVA was performed. $P$ values were calculated with a post hoc Bonferroni multiple comparison (only pairs relative to the untreated control simultaneously compared). Error bars: standard error. $n=3$ replicates per inhibitor per concentration.

DS brains, the third copy of BACE2 skews the antiamyloidogenic processing significantly towards BACE2cleavages, relative to other anti-amyloidogenic enzymes cleaving at the $\alpha$-site. Importantly, these CSF results validate the in vivo relevance of the peptide ratios obtained using CM from iPSC-derived cerebral organoids (comparison of Fig. 1b and Fig. 1c).

\section{A $\beta$-degrading activity of BACE2 is cross-inhibited by clinically trialed BACE1 inhibitors}

Chemical inhibition of BACE1 remains an attractive therapeutic strategy for AD. As BACE2 is a homologous protein, most inhibitors tested in clinical trials also cross-inhibit the (pro-amyloidogenic) $\beta$-secretase activity of BACE2, which has been proven as the cause of several unwanted side-effects, such as skin pigmentation changes. As our data suggest that the opposite, A $\beta$-degrading, activity of BACE2 has an important role, we designed a new FRET-based in vitro assay, in which efficient $\mathrm{A} \beta \mathrm{DP}$-cutting after $\mathrm{A} \beta$ aa34 by BACE2 at $\mathrm{pH}=3.5$ could be measured (Fig. 2), whereas zero activity by BACE1 was detectable under same conditions (Supplementary Information). We demonstrated that at least two BACE1 inhibitor compounds (of which one recently used in clinical trials) inhibit the $A \beta D P(A \beta-$ clearance) activity of BACE2 in a dose-dependent manner (Fig. 2). This has, to our knowledge, so far not been shown, and could provide an additional explanation for the failure of some BACE1 inhibitor clinical trials, and should be taken in consideration when testing new inhibitors.

\section{$A \beta D P$ product $(A \beta x-34)$ colocalises with BACE2 in human brain and organoid neurons}

As in vitro experiments showed that BACE2 can very efficiently cleave the A $\beta 34$ site in the FRET peptide (Fig. 2) 

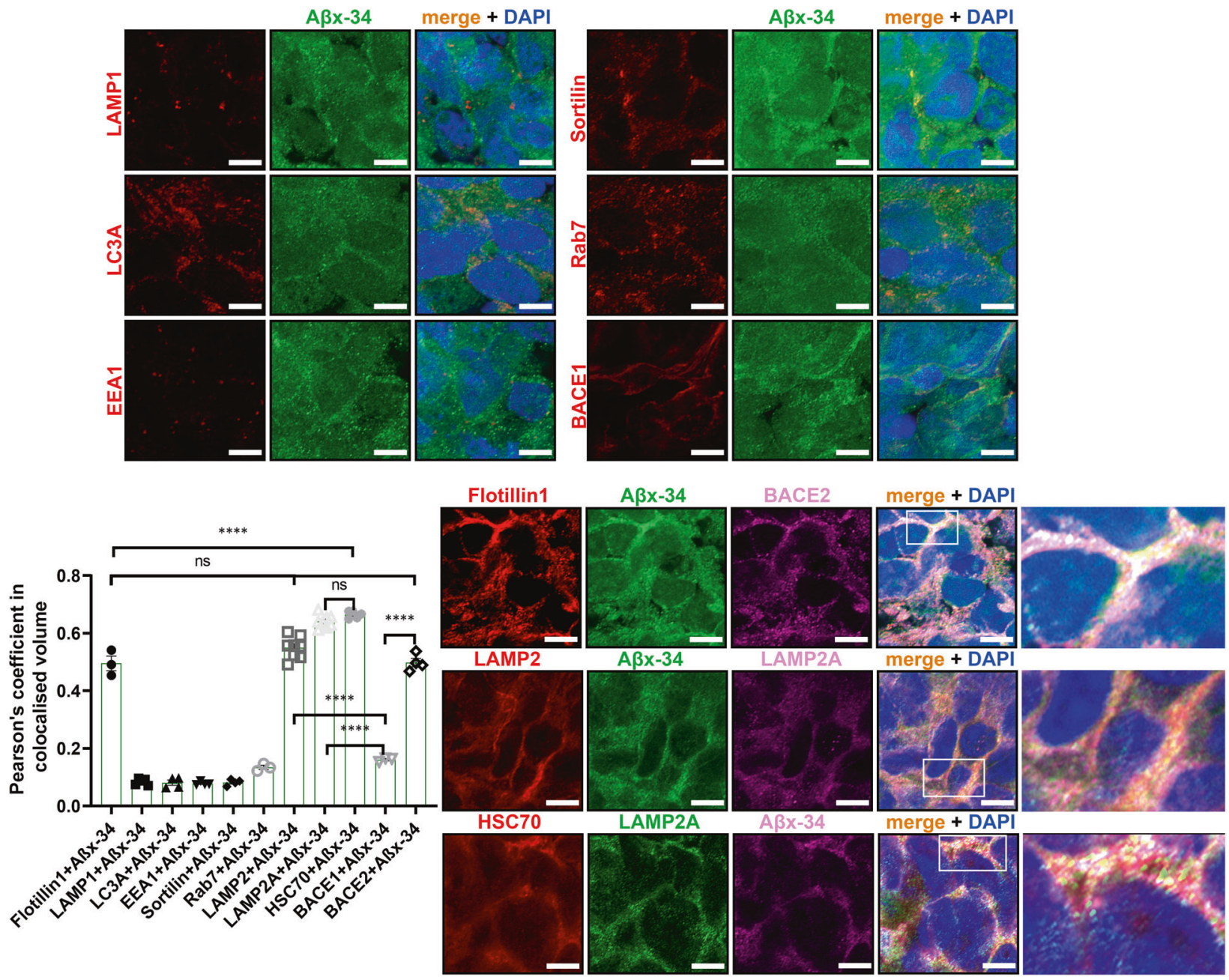

Fig. 3 Subcellular compartment localisation of $A \beta$ degradation product Aßx-34 in hiPSC-derived cerebral organoid sections. Pairwise Pearson's coefficient of colocalisation for a pair of co-stained antibodies: $\mathrm{A} \beta \mathrm{x}-34$, and a specific marker for the subcellular vesicle compartment: lipid rafts (Flotillin1), lysosomes (LAMP1), macroautophagosomes (LC3A), early endosomes (EEA1), macroautophagosome-lysosome fusion/exosomes (Sortilin), late endosomes (Rab7), specific sub-sets of lysosomes (LAMP2, LAMP2A) and CMA-chaperone (HSC70). In the final two columns of the histogram, the Pearson's colocalisation level was shown between $A \beta x-34$ and
BACE1 or BACE2, respectively (repeated in more detail in Supplementary Fig. 7). Greater than $95 \%$ of cells in all of these images were MAP2+ neurons (not shown). Representative images of the organoid stainings from which the coefficients were calculated are shown in the panels. Last column in the bottom right panel is the zoomed-in inset from the previous column. Images were captured using AiryScan Zeiss confocal microscopy, and single $0.16 \mu \mathrm{m}$ slices are shown (from $20 \mu \mathrm{m}$ full z-stack analysed). Error bars: standard error, $p$ values: after standard one-way ANOVA using post hoc Bonferroni multiple comparison calculation. Scale bar: $5 \mu \mathrm{m}$. and synthetic $\mathrm{A} \beta 1-40$ peptide in solution at an acidic $\mathrm{pH}$ [12], we sought to visualize if the presence of the substrate (A $\beta 1-40)$, enzyme (BACE2), and one of the products of this reaction (A $\beta 1-34)$ can be detected in our organoids, in a subcellular compartment known to be acidic. First, by immunofluorescence (IF) using pan-anti-A $\beta$ (4G8), antiBACE2, or neo-epitope-specific antibodies against $A \beta x-40$ and $A \beta x-34$ [23], we detected significantly higher signals (normalized to pan-neuronal marker) in T21 organoid neurons, compared with isogenic D21 ones (Supplementary Fig. 4b-d). Pearson's coefficient showed a high level of colocalisation $(>0.55)$ of both the main substrate $(\mathrm{A} \beta \mathrm{X}-40)$ and its putative degradation product $(\mathrm{A} \beta \mathrm{x}-34)$ with $\mathrm{BACE} 2$ in neurons of cerebral organoids, in LAMP2 + compartment (known to be a subset of lyzosomes, therefore low $\mathrm{pH}$ vesicles) (Fig. $3 \&$ Supplementary Fig. 7). In comparison, the Pearson's coefficient for BACE1 with $\mathrm{A} \beta \mathrm{x}-34$ was only 0.16 (Fig. $3 \&$ Supplementary Fig. 7), and its pattern of subcellular localization was different to BACE2 (high colocalization with Rab7 and Sortilin, much lower with LAMP2). Using IF on human brain sections, a similar highly significant difference was observed (Fig. 4a, b): A $\beta \mathrm{x}$ 34 colocalised with BACE2 $(0.52( \pm 0.034$ SEM $))$ as opposed to BACE1 $(0.01( \pm 0.021$ SEM $))$. The colocalised signal of $A \beta x-34$ and $B A C E 2$ was seen in three categories of objects (Fig. 4), in all analysed samples: four individual 

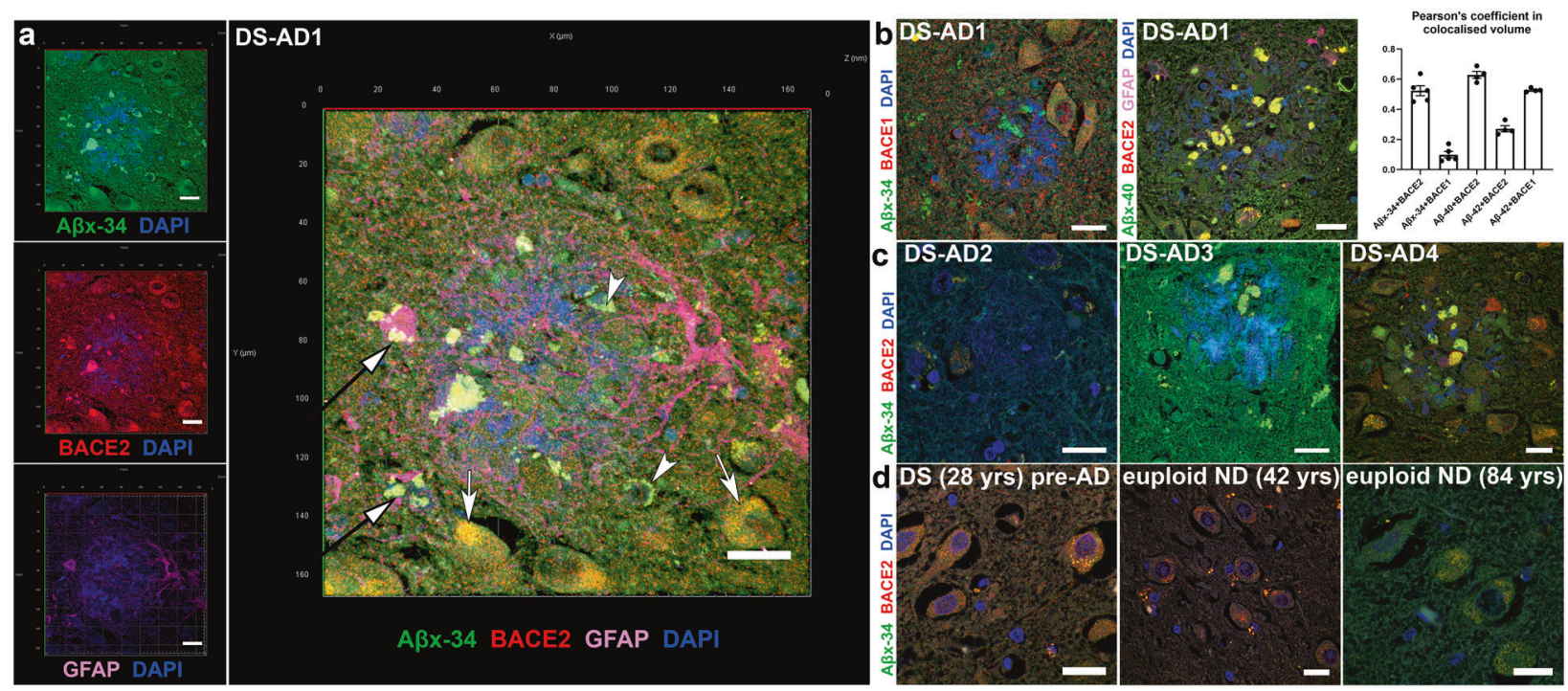

Fig. 4 Localisation of A $\beta D P$ degradation products and $\mathrm{A} \beta$ peptides with BACE2 in hippocampal sections of the human post-mortem brain. a Immunofluorescence analysis of the brain of DS-AD-1 costained for A $\beta \mathrm{x}-34$, BACE2, and GFAP. A typical near-circular neuritic plaque is shown (in which DAPI faintly stains the fibrillar amyloid deposits). Arrows indicate three categories of objects in which the colocalisation of BACE2 and the A $\beta D P$ product $A \beta x-34$ is observed. White arrows: intraneuronal fine-vesicular pattern; white arrowheads: large intraneuronal spherical granules (lipofuscin); black arrows with white arrowheads: amorphous extracellular aggregates. See Methods and Supplementary Fig. 8 for experiments controlling the extent of lipofuscin autofluorescence effects. b DS-AD1 brain co-stained for $\mathrm{A} \beta \mathrm{x}-34$ and BACE1, or $\mathrm{A} \beta \mathrm{x}-40, \mathrm{BACE} 2$ and GFAP, and Pearson's coefficient of colocalisation for proteins stained in parts ' $a$ ' and ' $b$ ', with the addition of the staining for $\mathrm{A} \beta \mathrm{x}-42$ neo-epitope (not shown). Error bars: SEM. c Same IF staining combinations as in part 'a' (except for GFAP) were used in three additional brain samples: DS-AD-2, 3, and 4. d Brain sample co-stained for BACE2 and A $\beta x-34$ of a 28 yrs old person with Down syndrome without dementia, and euploid nondemented (ND) controls aged 42 and 84 . Scale bar: $20 \mu \mathrm{m}$. a Elimination of the third copy of BACE2 by CRISPR-Cas9 in T21C5 hiPSC decreases ABDP/AB ratio in CM GTCTAGCACATACCGCTCCAAGGGCTTTGACGTCACAGTG AAGTACACACAAGGAAGCTGGACGGGCTTCGITGGGGAA GACCTCGTCACCATCCCCAAAGGCTTCAATACTTCTTTTCT TGTCAACATTGCCACTATTTTTGAATCAGAGAATTTCTTTTT CCACACTTGCCAAG

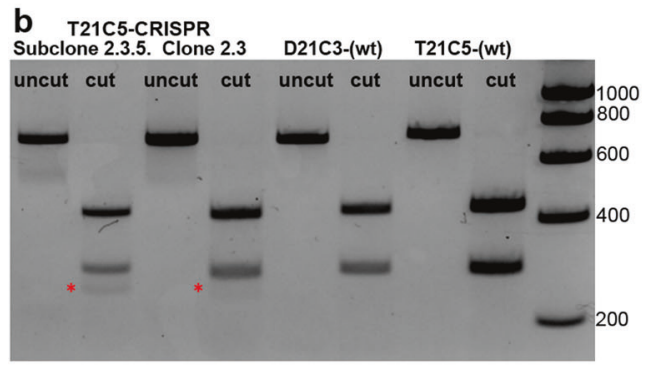

Fig. 5 CRISPR/SpCas9-HF1-mediated reduction of BACE2 copy number from three to two in the T21C5 hiPSC line. a $B A C E 2$ exon3 sequence with $7 \mathrm{bp}$ deletion (yellow) provoked by the CRISPR/ SpCas9-HF1 is shown. Red: restriction endonuclease HpyCH4IV sites (a de novo HpyCH4IV site is generated by the $7 \mathrm{bp}$ deletion). b agarose gel electrophoresis of the $733 \mathrm{bp}$ PCR product containing the targeted site before (uncut) and after digestion with HpyCH4IV (cut), for the initial clone 2.5, and its colony-purified sub-clone 2.3.5 (renamed further below as " $\Delta 7$ "). The $294 \mathrm{bp}$ fragment in 2.3 .5 is reduced to $65 \%$ of the wt value (normalized to the $439 \mathrm{bp}$ band), and a
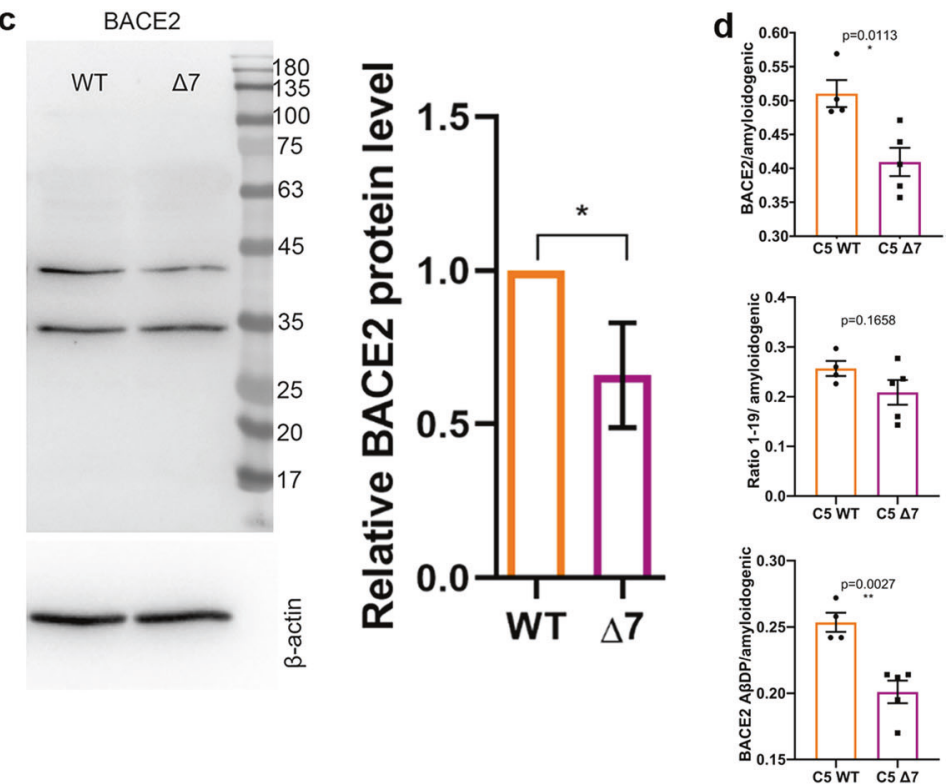

de novo $255 \mathrm{bp}$ fragment appears in CRISPR-targeted line (red asterisk). c Western blot stained with anti-BACE2 antibody of the lysates of the iPSC line $\Delta 7$ compared with the wt T21C5 iPSC line. Quantification of the total actin-normalised BACE2 signal showed a significant reduction in $\Delta 7$ compared with T21 unedited line. Error bars: standard error, $p$ value: student's $t$ test. d BACE2-A $\beta$ DP/amyloidogenic peptides ratio after IP-MS analysis of CM produced by the 48DIV organoids derived from the iPSC line $\Delta 7$ compared with the T21C5wt control were significantly decreased. Error bars: standard error, $p$ values: two-tailed $t$ test comparison. 

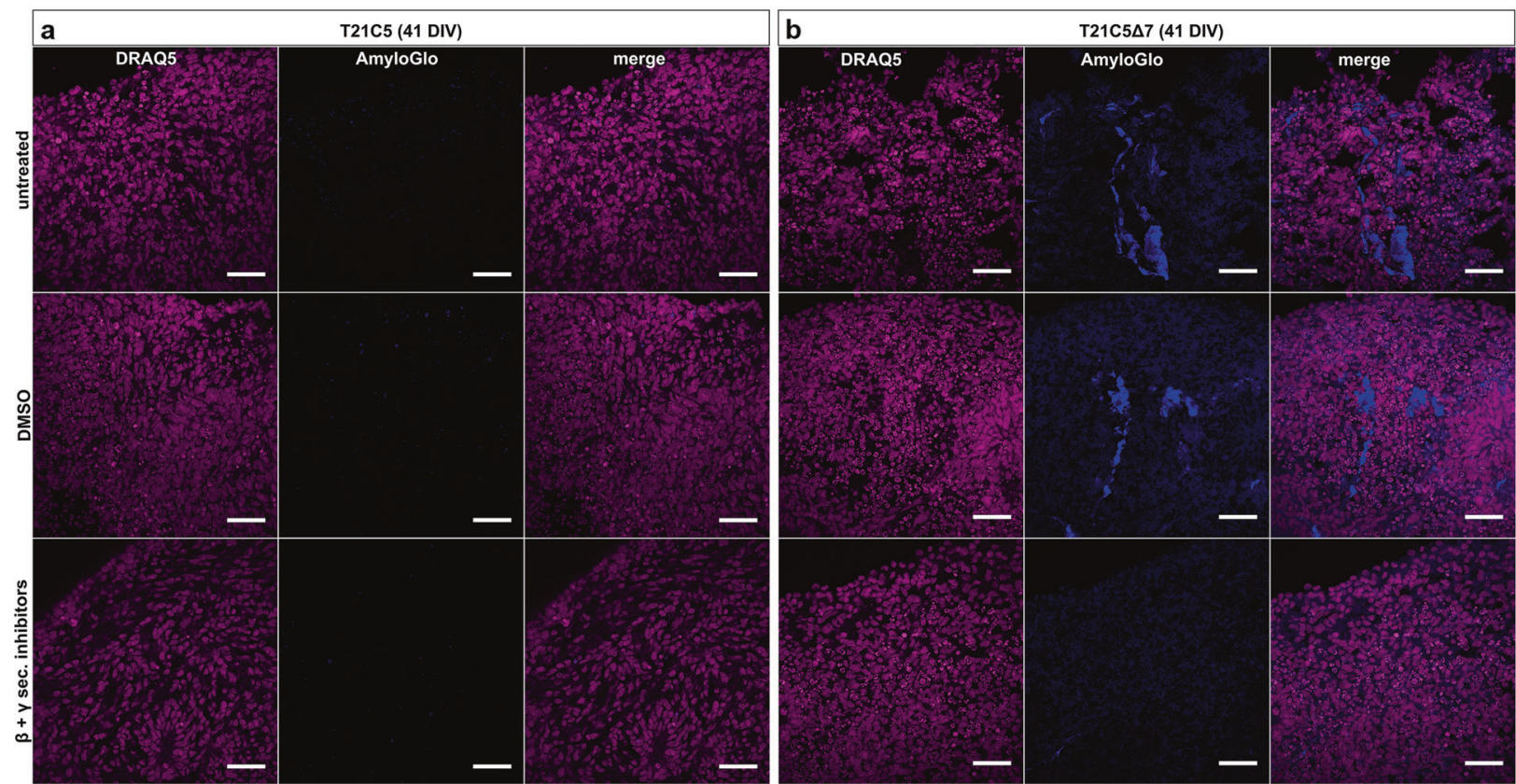
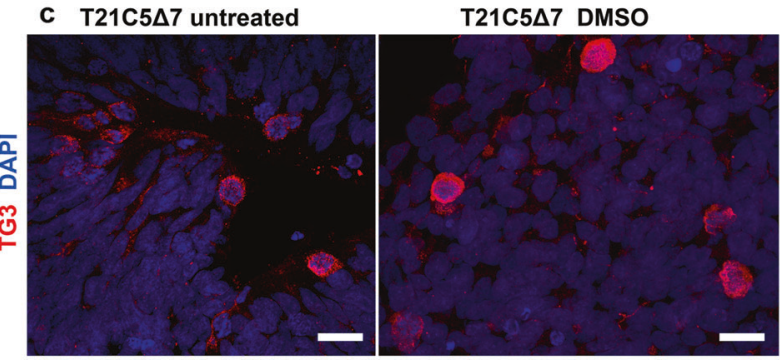

Fig. 6 CRISPR/SpCas9-HF1-mediated reduction of BACE2 copy number from three to two in the T21C5 hiPSC line provoked early AD-like pathology in organoids. a-c Early AD-like pathology was provoked in 41DIV T21C5 $\Delta 7$ organoids, but was not detected in T21C5 parental organoids. a-b Treatment of the T21C5 57 with combined $\beta \mathrm{I}$-IV ( $\beta$-sectretase inhibitor) and compound $\mathrm{E}$ ( $\gamma$-secretase inhibitor) from 20 to 41DIV completely prevented the formation of

DS-AD brains (Fig. 4a-c), five euploid sporadic AD subjects (example in Supplementary Fig. 8a, for complete list of brain samples see Supplementary Table 1) and (in the fine vesicle compartment only) in five non-demented control euploid subjects' neurons (age 42-84), as well as DS brain from a 28 yr old with no plaques or dementia, (examples in Fig. 4d, for complete list of brain samples see Supplementary Table 1). Lambda scanning and Sudan black B stainings were independently used to subtract the autofluorescence of lipofuscin granules (Supplementary Fig. 8f, g). This has proven that the fine-vesicular pattern and large amorphous extracellular aggregates are not autofluorescent lipofuscin granules, but real colocalisations of BACE2 and A $\beta \mathrm{x}-34$ (Supplementary Fig. 8). Colocalised signals of $\mathrm{A} \beta \mathrm{x}-34$ and BACE2 were particularly strong in areas surrounding neuritic plaques (Fig. $4 \mathrm{a}-\mathrm{c}$ ).
T21C5 $\Delta 7 \beta+y$ sec. inhibitors

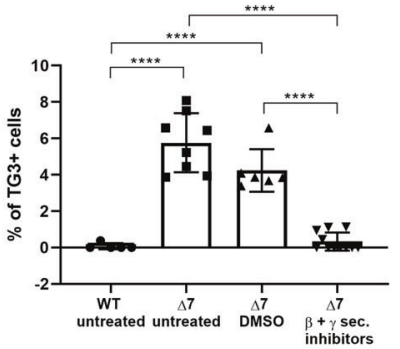

extracellular amyloid deposits. Staining with amyloid specific dye (AmyloGlo) and nuclear dye (DRAQ5). Scale bar $50 \mu \mathrm{m}$. c $\beta$ - and $\gamma$ secretase inhibitor treatment highly significantly reduced the presence of TG3 + (pathologically conformed Tau) cells in T21C5 57 organoids compared with untreated T21C5 7 organoids. Scale bar: $20 \mu \mathrm{m}$. Error bars: $\mathrm{SD}, * * * * p<0.0001$. Only statistically significant differences are shown.

As A $\beta D P$ cleavage by BACE2 is efficient only at low $\mathrm{pH}$, we sought to analyse in more detail the BACE2 and $\mathrm{A} \beta \mathrm{x}-34$ colocalisation in highly acidic cellular compartments. For this reason, we co-stained lysosome markers LAMP1 or LAMP2 with $\mathrm{A} \beta \mathrm{x}-34$. In addition, macroautophagic vacuoles containing $A \beta$ were shown to accumulate in $\mathrm{AD}$ distended neurites [24], which is why we also stained with the macro-autophagosome marker LC3A. As we further found that $\mathrm{A} \beta \mathrm{x}-34$ did not colocalise with LAMP1 or LC3A, but colocalised strongly with LAMP2 (Fig. 3, Supplementary Fig. 7 and Supplementary Information), we tested colocalisation with the components of an alternative autophagy pathway: chaperone-mediated autophagy (CMA), and found a very high level of colocalisation (Fig. 3). 

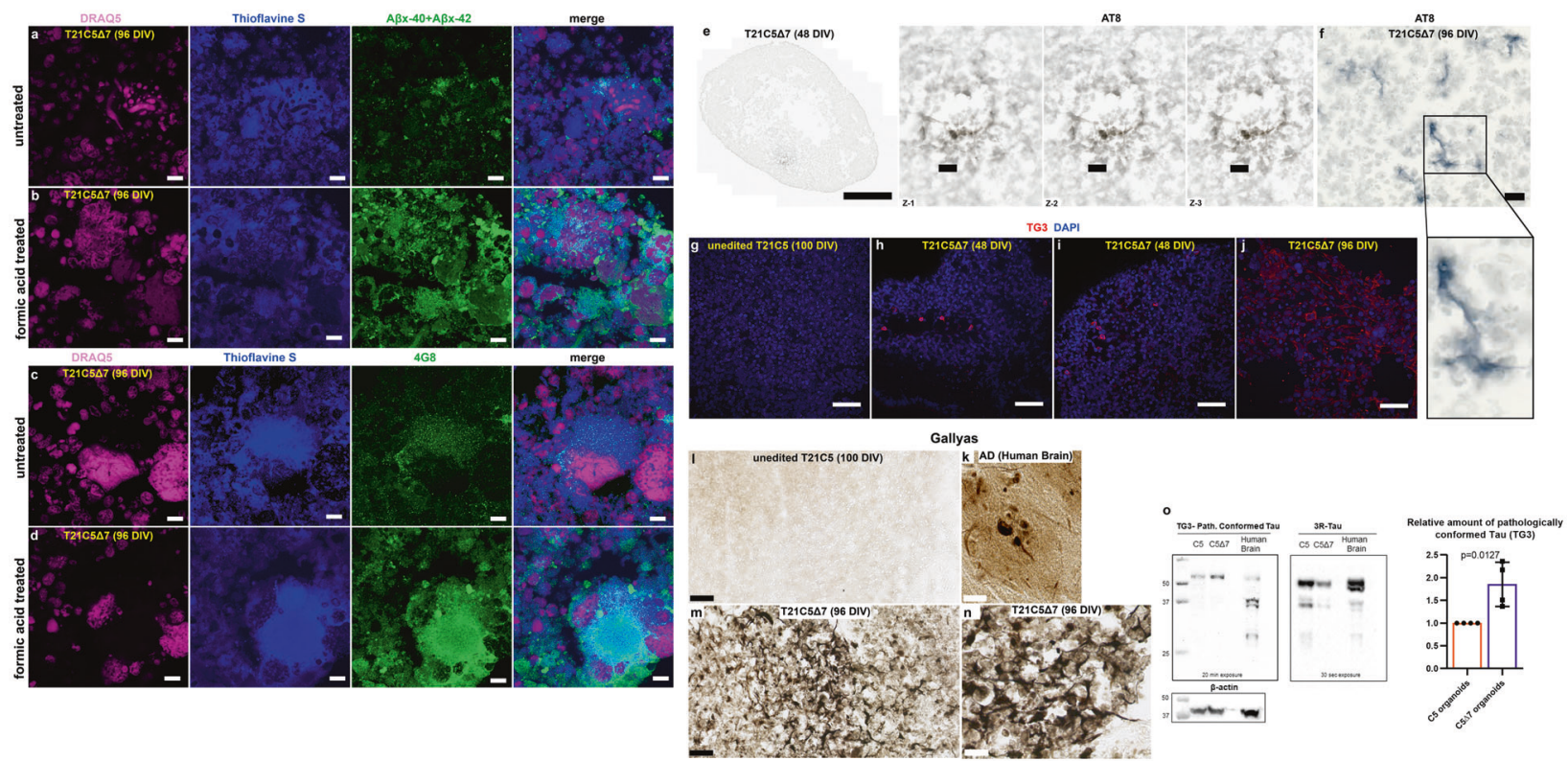

Fig. 7 Amyloid and Tau pathology are shown with six different methods in T21C5 77 organoids that have BACE2 copy number reduced from three to two by CRISPR/Cas9. a-d The signal of amyloid specific antibodies $\mathrm{A} \beta \mathrm{x}-40+\mathrm{A} \beta \mathrm{x}-42(\mathbf{a}, \mathbf{b})$, or $4 \mathrm{G} 8(\mathbf{c}, \mathbf{d})$ colocalising with Thioflavine $\mathrm{S}$ in T21C5 57 (96DIV) organoids was drastically increased upon treatment with $87 \%$ Formic acid for 10 minutes at RT, proving it contains the insoluble extracellular $\beta$ amyloid deposits. Scale bar: $10 \mu \mathrm{m}$. e AT8 (hyperphosphorylated Tau) positive neurites within plaque-like structure in 48DIV organoids. Left: the whole organoid slice, scale bar: $500 \mu \mathrm{m}$. Right: zoom in on the plaque-like structure from ' $\mathrm{e}$ ', in the three individual $\mathrm{z}$-slices (interval between slices, $1 \mu \mathrm{m}$; scale bar: $20 \mu \mathrm{m}$ ). f AT8 (hyperphosphorylated Tau) positive neurites in 96DIV organoids. Scale bar: 10 $\mu \mathrm{m}$. $\mathbf{g}-\mathbf{j}$ TG3 (conformationally altered Tau) staining of unedited control T21C5 (100DIV) g, CRISPR-edited T21C5 7 (48DIV) with TG3-positive neurons in 48DIV organoids $\mathbf{h}$, i, and CRISPR-edited

\section{Trisomy of $B A C E 2$ skews non-amyloidogenic $A \beta$ peptide ratios and suppresses $A D$-like pathology in organoids}

Using CRISPR/SpCas9-HF1, we eliminated a single copy of $B A C E 2$ in the trisomic iPSC clone C5 (T21C5 $\Delta 7$, a $\triangle 7 \mathrm{bp}$ in $B A C E 2$ exon3, knocking out one of three copies of the gene), while maintaining the trisomy of the rest of chromosome 21 (Fig. 5a-c, Supplementary Fig. 9, Supplementary Information). Total actin-normalised BACE2 signal showed a $27-34 \%$ reduction in $\Delta 7$ compared with $\mathrm{T} 21$ unedited clone, and no significant difference compared with D21 control (Fig. 5c, Supplementary Fig. 10). Total protein level of APP in $\Delta 7$ remained at trisomic levels, significantly increased compared with the disomic control (Supplementary Fig. 10). The CRISPR-edited iPSCs formed cerebral organoids expressing markers of all six neuronal layers by 48 days in vitro (DIV) (Supplementary Fig. 9c). The CRISPR correction of $B A C E 2$ gene dose from three to two, resulted in a significant decrease in levels of putative
T21C5 7 (96DIV) showing many TG3 + neurons with diffuse staining of extracellularized mal-conformed Tau aggregates $\mathbf{j}$. Scale bar: $50 \mu \mathrm{m}$. $\mathbf{k}-\mathbf{n}$ Gallyas staining of human $\mathrm{AD}$ brain $\mathbf{k}$, unedited control T21C5 (100DIV) l, CRISPR-edited T21C5 7 (96DIV) $\mathbf{m}$, n shows negative staining in parental unedited organoid $\mathbf{l}$ and very strong signal in neurons and plaque-like associated neurites within T21C5 $\Delta 7$ organoid $\mathbf{m}, \mathbf{n}$. Scale bars: $50 \mu \mathrm{m} \mathbf{l}, \mathbf{m}$ and $20 \mu \mathrm{m} \mathbf{n}$ and $5 \mu \mathrm{m} \mathrm{k.} \mathrm{o}$ Representative western blot of T21C5 and T21C5 7 organoid lysates stained using antibodies against pathologically conformationally altered Tau (TG3) or general 3 repeat ( $3 \mathrm{R})$ Tau. $\beta$-actin was used as a loading control. Human brain tissue of a 75 year old is shown for comparison. Comparison of the average values $(n=4)$ for CRISPRedited T21C5 57 showed a highly significant relative increase in TG3 compared with unedited $(n=4) \mathrm{T} 21 \mathrm{C} 5$ organoids, as indicated in the graph, $p=0.0127$.

BACE2-A $\beta \mathrm{DP}$ (A $\beta$-clearance) products $(1-20 \& 1-34)$, as well as total BACE2-related non-amyloidogenic peptides (1-19 \& 1-20 \& 1-34), relative to amyloidogenic peptides (Fig. 5d). This pinpoints the triplication of BACE2 as a likely cause of specific anti-amyloidogenic T21 effects we observed in Fig. 1a. Furthermore, we used two different dyes to detect any presence of amyloid deposits (the traditional Thioflavine $\mathrm{S}$, and a newer, more sensitive dye AmyloGlo [25]) in organoid sections. Remarkably, elimination of the third BACE2 copy caused the T21 organoids (that had not shown any overt amyloid deposits at 100DIV, see T21C5 in Supplementary Fig. 11, top row) to develop extremely early AD-plaque-like deposits (AmyloGlo+ and Thioflavine $\mathrm{S}+$ ) in the cortical part of the organoid by 48DIV (Supplementary Fig. 11, middle row), that progressed aggressively and became much stronger and denser by 96 DIV, accompanied by massive cell death (Supplementary Fig. 11, bottom row, Supplementary Fig. 12).

In order to prove that extracellular deposits staining positively with amyloid dyes really are related to 


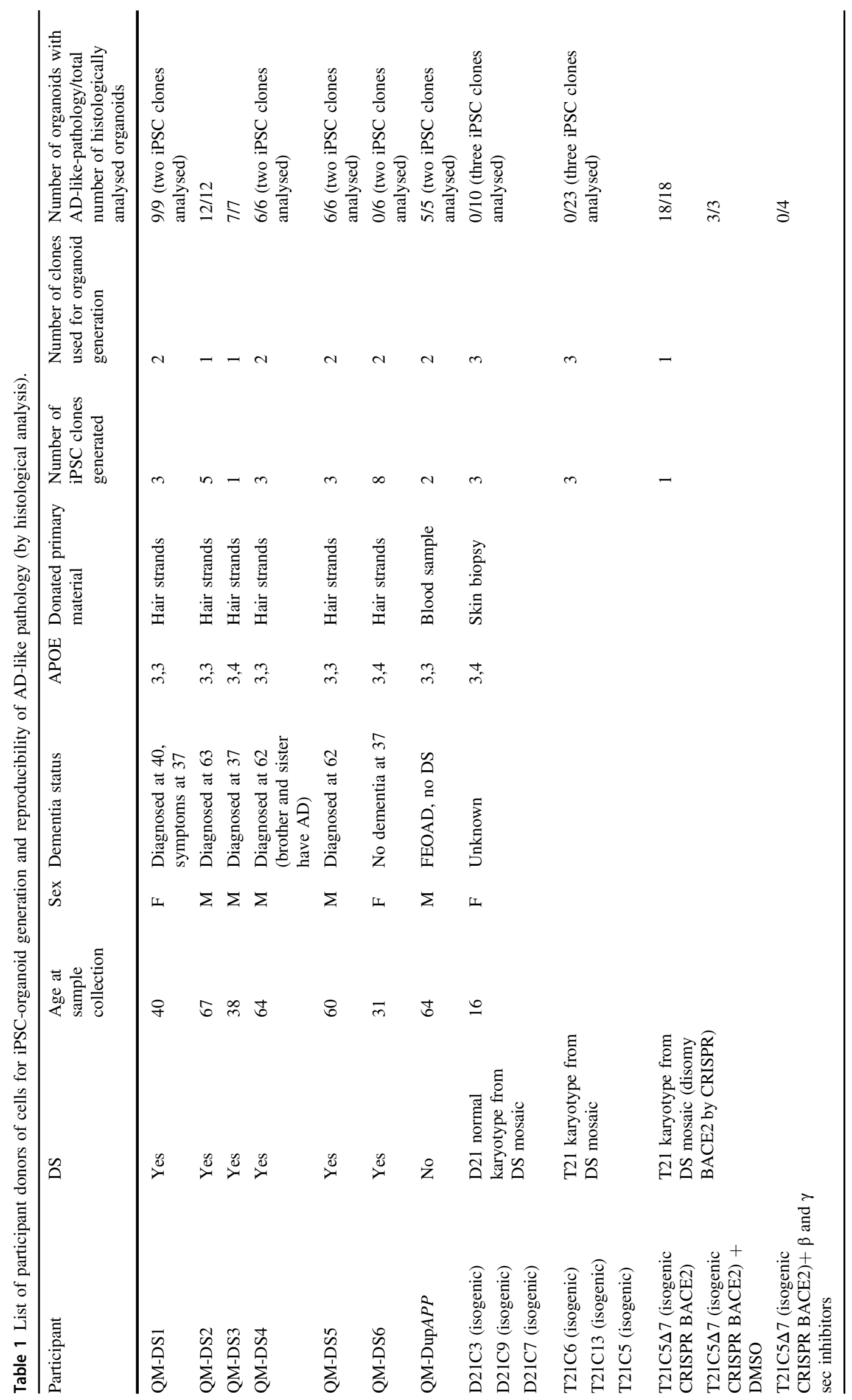




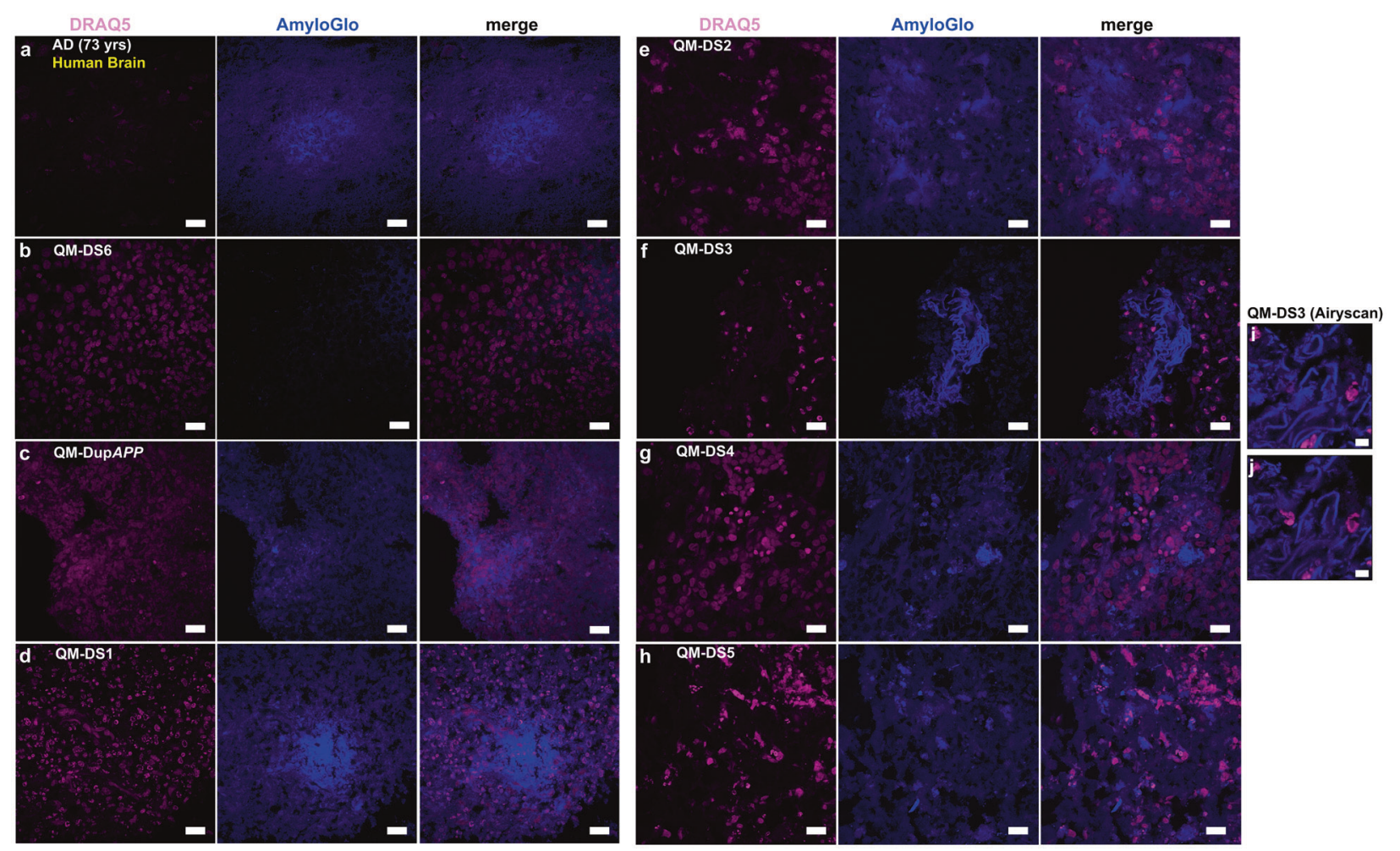

Fig. 8 Amyloid-like pathology, staining with AmyloGlo, is shown in different lines of organoids and Human AD Brain served as positive control. a Human $\mathrm{AD}$ Brain (73 yrs) shows amyloid plaques in the Entorhinal cortex. b QM-DS6 (DIV100) shows no AD pathology. c-h QM-DupAPP and QM-DS1, 2, 3, 4, 5 show
AmyloGlo-positive aggregates, similar to human brain. Scale bar: $20 \mu \mathrm{m}$. i, j Airyscan analysis of QM-DS3 showing super-resolution images of AmyloGlo-positive material with fibrillar-like appearance. Scale bar: $5 \mu \mathrm{m}$. hyperproduction of $\mathrm{A} \beta$ amyloidogenic peptides, we cultured T21C5 57 organoids in media containing high concentrations of $\beta$ and $\gamma$ secretase inhibitors. Early T21C5 and T21C5 57 organoids were treated with a combination of $\beta$ secretase inhibitor IV and compound E ( $\gamma$ secretase inhibitor XII) (Supplementary Table 2) from 20DIV to 41DIV (Fig. 6). Amyloid-like deposits were readily detected with AmyloGlo in the untreated and vehicle only treated T21C5 $\Delta 7$ organoids (Fig. 6b), but were completely absent from T21C5 $\Delta 7$ organoids treated with $\beta$ and $\gamma$ secretase inhibitors (Fig. 6b, bottom row). Inhibitor treatment also significantly reduced the number of neurons expressing pathologically conformed Tau (TG3-positive cells) in the T21C5 47 compared with untreated controls (Fig. 6c). No AmyloGlo-positive aggregates or TG3-positive cells were detected in T21C5 organoids under any treatment conditions at DIV41 (Fig. 6a, c) and were also absent in the same organoids at DIV100 (Fig. 7g, 1, Supplementary Fig. 11). Also, no obvious deleterious effects of the inhibitors, or vehicle control, could be seen in early unedited T21C5 organoids.

Further histo-pathological verification showed that elimination of one copy of BACE2-triggered progressive accumulation of extracellular deposits that co-stain with Thioflavine $\mathrm{S}$ and antibodies against $\mathrm{A} \beta$, both $4 \mathrm{G} 8$ and neoepitope specific $A \beta x-40 \& A \beta x-42$. The antibody signal intensity in colocalisations with Thioflavine $\mathrm{S}$ drastically increased upon pre-treatment with $87 \%$ formic acid (Fig. 7a-d), proving that the deposits contain insoluble $A \beta$ material. This is further corroborated by the isolation of fibrillary material from the detergent-insoluble fraction of the CRISPR-edited organoid. When viewed by transmission electron microscopy (TEM) the filaments found exhibited a straight morphology of $<10 \mathrm{~nm}$ diameter (Supplementary Fig. 13a), closely resembling fibrils grown in vitro from synthetic A $\beta 1-40$ peptide (Supplementary Fig. 13c). Furthermore, neuritic plaque-like features were detected by IHC co-staining with Gallyas in CRISPR-edited organoids (Fig. 7m, n), but not their unedited T21 control (Fig. 71). Human brain from an AD patient is shown for comparison stained with Gallyas (Fig. 7k). Tau pathology was also observed by IHC using the hyperphosphorylated Tau antibody AT8 (Fig. 7e, f), and by IF for conformationally altered Tau (TG3, Fig. $7 \mathrm{~g}-\mathrm{j}$ ). The relative increase in the amount of conformationally altered (pathological) Tau in CRISPR-edited organoids T21C5 7 , compared with 


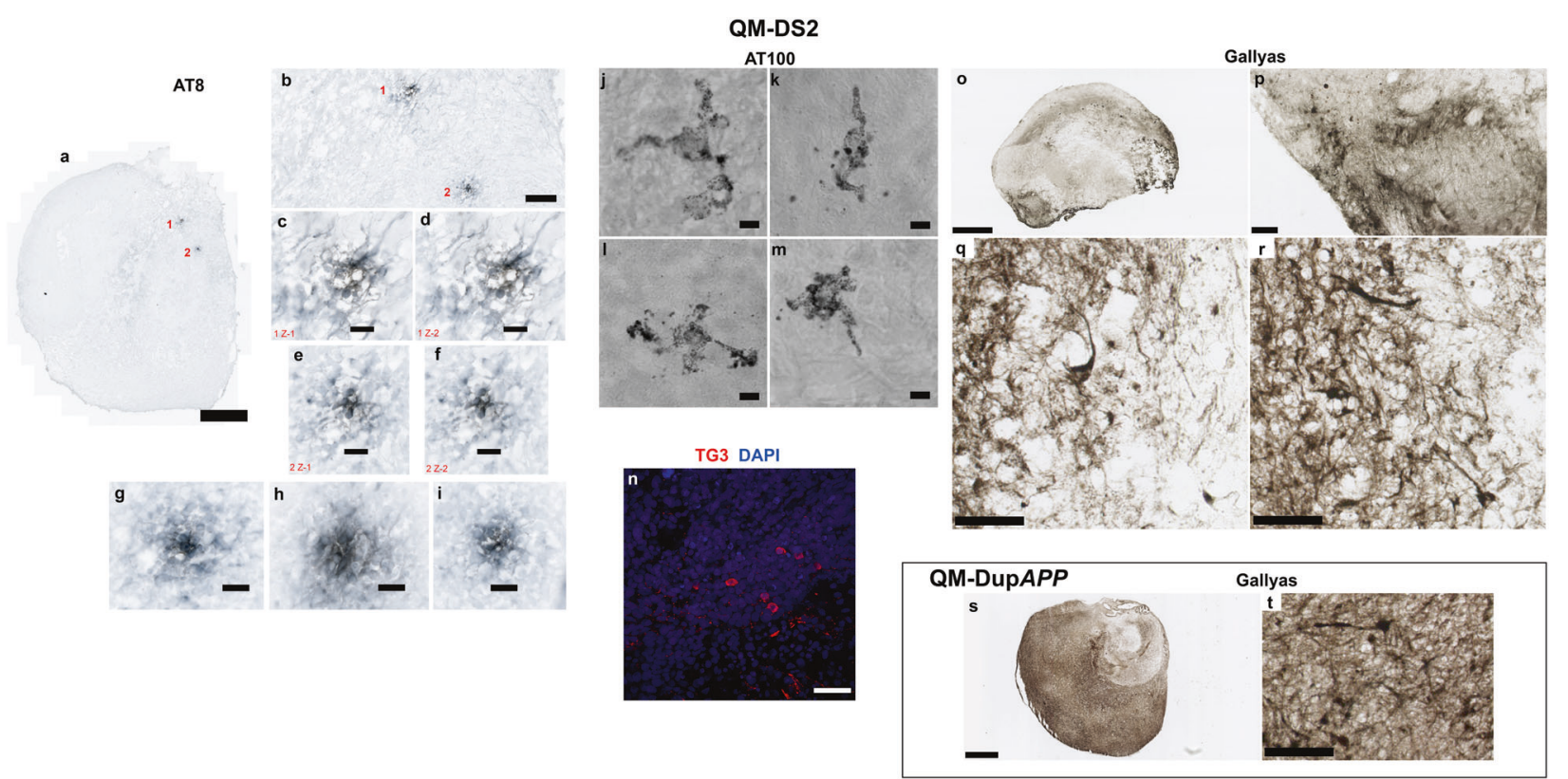

Fig. 9 Tau pathology, staining with Gallyas, and with three different antibodies (hyperphosphorylated, conformationally altered, and filamentous Tau) in QM-DupAPP (100DIV) and QM-DS2 (100DIV) organoids. a Scan of whole QM-DS2 organoid section shows two hyperphosphorylated Tau foci (neuritic plaque-like structures). Scale bar: $500 \mu \mathrm{m}$. b Zoom in on the same foci. Scale bar: 100 $\mu \mathrm{m}$. c, d AT8-positive neurites in the pathological structure number 1 in two individual z-slices. Scale bar: $20 \mu \mathrm{m}$. e, f AT8-positive neurites in the pathological structure number 2 in two individual z-slices. Scale bar: $20 \mu \mathrm{m}$. g-i AT8-positive neurites in further three pathological foci found at a different depth, from the same organoid (not shown at lower

unedited T21 control organoids, was also independently confirmed by immunoblotting using TG3 antibody. As shown in Fig. 7o, the protein material isolated from T21C5 57 organoids produced significantly more TG3 signal than unedited controls, albeit having a weaker signal with the general 3R-Tau antibody (consistent with the observed neuronal loss, Supplementary Fig. 12).

\section{AD-like pathology develops reproducibly in unedited cerebral organoids from $71 \%$ of DS donors, and it is donor-specific}

Our data in Figs. 5-7 show that severing the BACE2 dose by a third, using CRISPR/Cas9, might tip the balance against the anti-amyloidogenic activity, and provoke $\mathrm{AD}$ like pathology. Our data in Fig. 1 suggest that antiamyloidogenic activity of BACE2 is gene dose dependent, and its level varies between individuals, and it has been previously reported that SNP allelic differences in BACE2 gene correlate with age of dementia onset in DS [26]. We therefore hypothesized that organoids grown from some people with DS may develop AD-like pathology without any CRISPR-Cas9 intervention. We then tested this magnification). Scale bar: $20 \mu \mathrm{m}$. j-m AT100 (filamentous Tau) positive neurons in the cortical layer of QM-DS2 organoid, partly showing "ballooned neuron" pathology. Scale bar: $5 \mu \mathrm{m}$. n TG3 (conformationally altered Tau) positive cells in the cortical layer of QM-DS2 organoid. Scale bar: $50 \mu \mathrm{m}$. o Scan of whole QM-DS2 organoid section stained with Gallyas. Scale bar: $500 \mu \mathrm{m}$. p-r zoom in on the parts of the same organoid shows strongly Gallyas-positive individual neurons. Scale bar: $50 \mu \mathrm{m}$ p $20 \mu \mathrm{m}$ q, r. s Scan of whole QM-DupAPP organoid section stained with Gallyas. Scale bar: 500 $\mu \mathrm{m} . \mathbf{t}$ Zoom in on the same organoid shows equally strong individual neurons as in QM-DS2 organoid. Scale bar: $50 \mu \mathrm{m}$.

hypothesis using iPSC lines from six different individuals with DS, and one DupAPP patient (Table 1). We detected amyloid-like aggregates (both diffuse and compact in appearance) in $5 / 7$ unedited iPSC-derived organoids from people with DS, and one with DupAPP (Fig. 8). The two donors whose iPSC-organoids did not show pathology are (i) the T21 iPSC from our isogenic model (whose clinical status is unknown) and (ii) QM-DS6, a donor who remains free from dementia symptoms at age 37 (Table 1). Organoids from another five DS donors, and one DupAPP patient, (all diagnosed with clinical dementia) all showed presence of diffuse and compact amyloid-like deposits (Fig. 8) as well as presence of neuritic plaque-like features (focal hyperphosphorylated tau (AT8+), conformationally altered tau (TG3+), and filamentous Tau (AT100+)) within neuropil neurites within plaque-like circular foci (Fig. 9a-n). This was corroborated by Gallyas intraneuronal positivity (Fig. 9o-t). Similarly as for T21C5 $\Delta 7$, we were able to isolate fibrillary material from the detergentinsoluble fraction of QM-DupAPP organoid (Supplementary Fig. 13b), that on TEM resembled fibrils grown in vitro from synthetic A $\beta 1-40$ peptide (Supplementary Fig. 13c). Most importantly: tested individual organoids from one 
donor (from multiple iPSC clones and multiple independent experiments) either all did (DupAPP, QM-DS1-5), or all did not (isogenic T21, QM-DS6) show AD-like pathology (Table 1), proving the pathology is donor dependent. This open possibilities of developing assays for pre-therapy riskstratification and individualized drug-response quantitation. In order to further test the hypothesis that decreasing the level of BACE2 may provoke some AD-like pathology in organoids, we artificially decreased the level of BACE2 using shRNA, in the QM-DS6 patient's iPSCs, that did not show AD-like pathology. The shRNA succeeded in decreasing the BACE2 protein level by only $28 \%$, (Supplementary Fig. 14a) similar to the CRISPR correction of three to two copies shown in Fig. 5. This intervention indeed provoked early (46DIV) presence of AmyloGlo+ aggregates, and a significant increase in TG3+ neurons (showing pathologically conformed Tau), (Supplementary Fig. 14b,c). Therefore, we conclude that correction of the trisomic over-expression of BACE2 in two different and independent individuals with DS (one using CRISPR, one using shRNA) both provoked a similar early AD-like pathology.

\section{Discussion}

Several human brain studies show detectable expression and $\beta$-secretase activity of BACE2, though at much lower levels than that of BACE1 [27-30]. Chemical inhibition of $\beta$-secretase activity is an attractive therapeutic approach aimed at reducing the production of $A \beta$ [31-33]. Complete knockout of Bacel abolished all $\beta$-secretase activity in mouse neurons, while leaving some degree of $\beta$-secretase activity in astrocytes [34]. This activity was abolished by the complete knockout of both Bace1 and Bace2, leading to a hypothesis that a BACE2-driven $\beta$-secretase activity in astrocytes may contribute to accelerate the $A \beta$-production and $\mathrm{AD}$ pathology in DS [34]. In human brain, the $\beta$ secretase activity of BACE1 correlated positively with the amount of $\mathrm{A} \beta$, whereas the $\beta$-secretase activity of BACE2 did not [27]. On the other hand, SNPs at the BACE2 locus (and not $B A C E 1$ ) correlate with the age of onset of dementia in people with DS [26], as well as sporadic LOAD in euploid people in the Finnish population [35], and a recent report showed that a de novo intronic deletion within one allele of BACE2 caused EOAD in a 50-year-old euploid person [36].

All of the above data implicate that a single allele alteration in the genetic dose of BACE2 is capable of affecting the risk of $\mathrm{AD}$ dementia, but do not resolve the question whether BACE2 per se acts predominantly as an accelerator, or a suppressor of AD pathology. The answer to this question requires clarification, as most chemical inhibitors used in clinical trials have dual activity against BACE1 and BACE2 [32, 37].

The increased ratios of $1-20 \& 1-34$ (BACE2-A $\beta D P$ ) to the amyloidogenic and $\alpha$-site products are among our most consistent and robust observations in T21 organoid CM and DS-CSF (Fig. 1b, c). The 1-34 generating cleavage can only occur after the cuts by both $\beta$ - and $\gamma$-secretases have released $A \beta$, because the hidden transmembrane site between aa34 and aa35 is inaccessible to any proteolytic enzymes until the soluble $A \beta$ (1-37 to 1-42) molecules are released from the membrane $[12,13]$. Therefore, the $A \beta 1$ 34 species can only be a product of an A $\beta D P$ activity (a catabolic degradation or clearance of an already made A $\beta 1-37$ to 1-42 peptides). Besides BACE2, the only enzymes with potential to cleave the peptide bond Leu34-Met35 are BACE1 [12, 14], and extracellular matrix (ECM) metalloproteinases (MMP2 and MMP9) [38], since no other $A \beta$-degrading enzymes (neither IDE, nor NEP, nor ECE) are known to cleave at this site [39]. BACE1 action is unlikely to cause the increased ratios we observe, as BACE1 can only generate this cut in solution at very high enzyme concentration and after prolonged incubation [12].

To further corroborate this point, we designed a novel FRET-assay and established the conditions in which BACE2 can efficiently cleave at A 34 site (Fig. 2) in 2 hours, conditions under which BACE1 activity at the A $\beta 34$ site was undetectable (Supplementary Information). We also demonstrated that two BACE1 inhibitors ( $\beta$ Secretase Inhibitor IV-CAS 797035-11-1 (Calbiochem, originally a Merck compound)), and LY2886721 (Eli Lilly compound recently used in clinical trials) both inhibit the A $\beta$ DP activity of BACE2 in vitro, whereas the $\gamma$-secretase inhibitor (DAPT) had no effect.

This suggests that the A $\beta D P$ activity (cutting the peptide bond Leu34-Met35) has a different enzymatic preference, conditions, and $\mathrm{pH}$, as compared with the classical $\beta$ secretase cleavage that both BACE1 and BACE2 are capable of. As FRET assays cleaving this classical (before Asp1) site are generally used to measure the BACE1 inhibitors' selectivity for BACE1 or BACE2, our data suggest that the degree of selectivity for any given inhibitor calculated this way, does not necessarily reflect whether the same selectivity would apply for their cross-inhibition of the Leu34-Met35 site cleavage (A $\beta D P$ ) activity. Interestingly, the presence of the $A \beta x-34$ degradation product, both alone [23] and colocalising with BACE2 (Fig. 4) show elevated levels in cells and extracellular aggregates immediately surrounding neuritic plaques, suggesting BACE2 degradation of not only newly produced $A \beta$, but also of $A \beta$ that is released and re-deposited (from and to) existing deposits. A recent report on widespread somatic changes in individual neurons suggests an additional mechanism for the production of toxic $A \beta$ species, including products that do not 
require secretase cleavage [40], underscoring the importance of efficient $A \beta$ degrading mechanisms that protect from $\mathrm{AD}$, such as the one exerted by BACE2 that we describe here.

A recent mouse model has shown that introducing a third dose of chromosome 21 to a mouse that several hundred fold overexpresses $A \beta 40$ and 42 worsens the amyloid plaque load, and this correlates with an unexpected decrease in the $A \beta 40 / 42$ ratio [41]. This unfavourable ratio effect (the cause of which is unknown) is expected to worsen the plaque load and AD pathology, and a mere $1.5 \times$ increase of Bace 2 dose in this mouse model has no chance in protecting the mouse against a $>100 \times$ overload of $A \beta$. In another mouse model, where transgenic $B A C E 2$ was artificially over-expressed together with transgenic wt $A P P$, it actually decreased $A \beta 40$ and 42 to the wt mouse control levels, and the presence of BACE2 transgene reversed behavioural pathologies seen in $\operatorname{Tg} A P P$ mouse [42].

This indicates that a balance of doses of $A P P$ and BACE2 affects levels of soluble $A \beta 40$ and 42 , and their oligomerization and aggregation as a consequence. Our results in Figs. 5-7 further corroborate that a significant disturbance of this balance by a reduction in BACE2 copy number is sufficient to cause an early AD-like pathology in T21 cerebral organoids. We did not see any amyloid plaque-like structures at $>100$ DIV organoids from three independent T21 iPSC clones (or normal disomic clones) of our isogenic system (Supplementary Figs. 1, 2, 4, 7, 11, 15, Figs. 3, 7, 8). Surprisingly, CRISPR/Cas9 elimination of the third copy of BACE2 in the same T21 clone caused widespread AmyloGlo+ deposits at 41DIV, and widespread neuritic plaquelike structures with profound neuron loss (Supplementary Figs. 11, 12) and Tau pathology at 96DIV (Figs. 6, 7). Our data in Fig. 1 suggest that anti-amyloidogenic activity of BACE2 is gene dose dependent, and its level varies between individuals, with SNP allelic differences in BACE2 correlating with age of dementia onset [26]. We therefore hypothesized that organoids grown from some people with DS may develop AD-like pathology without any CRISPR-Cas9 intervention. Diffuse amyloid plaque-like appearance with Tau pathology was recently reported in 110 days old cerebral organoids from only a single clone of a single DS-hiPSC line [43] so far. We subsequently analysed iPSC-derived organoids at approximately the same cell culture age from a total of seven different individuals with DS and one with DupAPP. We found flagrant AD-like pathological changes in $5 / 7$ DS tested $(71 \%)$, as well as the one DupAPP. Very interestingly, when this assessment was repeated in independent experiments, and when individual organoids from a single experiment were compared, it was a black/white picture: either they all had AD-like pathology, or none did, driven solely by the genotype of the donor (Table 1). Our data, though not conclusive, are illustrative of the stratifying potential of this technology. For example, the cerebral organoids from individual QM-DS3 showed the worst ADlike pathology with fibrillary amyloid deposits (Fig. 8f, i, j, Table 1), and this individual was diagnosed with dementia at age 37. In contrast, organoids from individual QM-DS6 showed no pathology (Fig. 8b, Table 1), and this individual was also dementia free at age 37 . This opens up possibilities for finding correlations with clinical parameters, for which a much larger number of individuals would have to be tested. Concordantly with our hypothesis, when QM-DS6 iPSCs had their BACE2 level lowered by $28 \%$ using an shRNA construct, this provoked early amyloid and Tau pathology in otherwise pathology-negative organoids (Supplementary Fig. 14).

To confirm that the AmyloGlo deposits were in fact aggregated $\beta$-amyloid containing material, early organoids were treated with a combination of $\beta$-secretase inhibitor IV $(\beta I-I V)$ and gamma secretase inhibitor XII (Compound E) (Fig. 6a, b). The combination of these inhibitors should prevent any production of $\mathrm{A} \beta$, and therefore eliminate AmyloGlo positivity. After treatment for 21 days, the inhibitor treatment did indeed prevent the formation of plaque-like deposits within T21C5 57 organoids, confirming that such deposits are comprised of $\beta$-amyloid. The same treatment conditions also significantly reduced the number of TG3-positive cells in T21C5 47 organoids (Fig. 6c), highlighting the ability to modulate both amyloid and tau pathology in the cerebral organoid system. This also demonstrates the feasibility of using this AD-like organoid pathology in future hypothesis-free drug screens for chemical compounds that may prevent/inhibit amyloid production or aggregation.

In view of our results, it becomes inviting to hypothesize that triplication of BACE2 may be the cause of the delayed onset of dementia in $30 \%$ of people with DS compared with DupAPP [7], and (because of the predicted abundance of BACE2 mRNA in endothelial cells) also the cause of a significantly lower degree of cerebral amyloid angiopathy (CAA) in the brains of people with DS compared with those of DupAPP [44]. Our organoid system is not informative in this regard, as we could not detect any endothelial cells in our organoids (not shown). This, however, is also an advantage, as it allows uncovering the mechanisms that are specific to neurons in the absence of endothelial or blood cell-derived tissue components.

In neurons, a recent report also found that an increased $A P P$ dose may act (through an unknown mechanism) as a transcriptional repressor of several chromosome 21 genes, including BACE2 [45]. This observation needs further verification and mechanistic explanation, but if true, it would imply that the protective effect of the third copy of $B A C E 2$ in DS that we observe is actually quenched by the third copy of $A P P$, which opens up possibilities of 
chemically intervening to inhibit this transcriptional repression and potentially unleash a much greater degree of BACE2 protection. An integration of the two observations (the one in [45] and the one in our report) suggests this could be exploited as an additional new protective/therapeutic strategy for $\mathrm{AD}$ in general.

We found, surprisingly, an equally high or higher level of colocalisation of A $\beta x-34$ with LAMP2A, as with the general LAMP2 (Fig. 3). The high level of colocalisation with LAMP2A and absence of colocalisation with either LC3A or LAMP1 (Fig. 3) suggest that A $\beta$ DP activity of BACE2 that generates A $\beta 34$ is not related to classical lysosomal degradation or macroautophagy, but rather could be related to a CMA-like process $[46,47]$. The only published study that linked CMA with APP processing [48] found a motif that satisfies the criteria for a CMArecognition KFERQ motif at the very C-terminus of APP (KFFEQ), and this paper demonstrated that C99 ( $\beta$-CTF) can bind HSC70. However, paradoxically, when this motif is deleted from the $\beta-\mathrm{CTF}$, the binding to HSC70 is not abolished, but rather increased, suggesting the presence of another, alternative CMA-recognition motif within the $\beta$ CTF peptide [48]. The association of the A $\beta D P \times-34$ product with LAMP2A/CMA compartment is a provocative new observation that requires further studies.

In conclusion, we found that relative levels of specific non-amyloidogenic and $\mathrm{A} \beta \mathrm{DP}$ (A $\beta$-clearance) products are higher in T21 organoids and DS-CSF, and they respond to the dose of BACE2 (and not APP). We also demonstrated that BACE2-A $\beta$ DP activity generating one of these products can be cross-inhibited in solution by recently clinically tested BACE1 inhibitors. All components of the A $\beta \mathrm{DP}$ degradation reaction (hitherto only demonstrated in solution in vitro): the main substrate $(\mathrm{A} \beta \mathrm{x}-40)$, the enzyme (BACE2), and its putative degradation product $(\mathrm{A} \beta \mathrm{x}-34)$, we found highly colocalised in discrete intracellular vesicles in human brain neurons, (and not astrocytes), suggesting that at least some of the $\mathrm{A} \beta \mathrm{DP}$ activity generating $\mathrm{A} \beta \mathrm{x}-34$ takes place intra-neuronally and physiologically during lifetime, before the onset of AD pathology, in both normal and DS brains. Furthermore, we directly demonstrated that the trisomic level of BACE2 protected T21-hiPSC organoids from early AD-like amyloid plaque pathology, therefore proving the physiological role of BACE2 as an ADsuppressor gene. The BACE2's $\theta$-secretase anti-amyloidogenic cleavage and the A $\beta D P$ degradation actions could both be contributing to an overall $\mathrm{AD}$-suppressive effect. Regardless of the contribution of each of these modes of action, our combined data suggest that increasing the action of BACE2 could be exploited as a therapeutic/protective strategy to delay the onset of $\mathrm{AD}$, whereas crossinhibition of BACE2-A $\beta$ DP activity by BACE1 inhibitors would have the unwanted worsening effects on disease progression. We also show that cerebral organoids from genome-unedited iPSCs could be explored as a system for pre-morbid detection of high-risk population for $\mathrm{AD}$, as well as for identification of natural dose-sensitive $\mathrm{AD}$ suppressor genes.

\section{Methods}

\section{Human subjects-clinical assessment}

Human subjects were participants in the "The London Down Syndrome Consortium (LonDownS): an integrated study of cognition and risk for AD in Down Syndrome" inter-disciplinary study, enrolled after informed consent, as per ethical approval 13/WA/0194, IRAS project ID:120344 see http://www.ucl.ac.uk/london-downsyndrome-consortium). Dementia diagnostic status was obtained via carer report and medical records, based on assessment by the individual's own clinician. To confirm diagnoses, we collected detailed data on dementia symptoms from the Cambridge Examination for Mental Disorders of Older People with Down's Syndrome and Others with Intellectual Disabilities (CAMDEX), a clinical tool used for diagnosing dementia in individuals with DS. This was independently reviewed by two psychiatrists. One iPSC line (QM-DS1) was established from an individual 40 year old who was diagnosed with dementia aged 40 . Consensus ratings agreed that this individual showed signs of dementia and died 2 years later following significant decline. AD was noted as the cause of death. Another line came from an individual (QM-DS2) who was diagnosed with dementia aged 63. The sample was taken at age 67 and at this time point, consensus was that the individual presented with signs indicative of possible dementia. This individual was still alive for follow up 2 years later, with a confirmed diagnosis of AD-dementia and AD-related seizures developed at age 68. The third line came from an individual diagnosed with dementia at age 37 (QM-DS3). Another line came from the individual QM-DS6, who donated hair at age 31 , and remains dementia free after follow up at age 37 . The age, sex, ApoE genotype, and dementia status of all other individuals are detailed in Table 1.

\section{Primary hair follicle keratinocyte sampling}

Upon specific informed consent, three to six individual strands of hair were non-invasively plucked from the scalp hair of donor subjects, and placed in transport medium (DMEM (Sigma D5546), 2 mm glutamine (Sigma G7513), 1x Pen/Strep (Sigma, P4333), 10\% foetal calf serum). Upon arrival to the laboratory, hair follicles were placed in collagen coated T25 flasks in KGM2 medium (Lonza 
CC-3107) and incubated at $37{ }^{\circ} \mathrm{C}, 5 \% \mathrm{CO}_{2}$. Primary keratinocyte cultures were split after reaching $35-50 \%$ confluency using $0.05 \%$ Trypsin/0.02\% EDTA.

\section{Reprogramming of primary keratinocytes}

Primary keratinocyte cultures were expanded to $70 \%$ confluency, electroporated with plasmids encoding reprogramming factors in episomal vectors (non-integrational reprogramming), and transferred to $0.1 \%$ gelatin coated wells (six-well plate), pre-seeded with mitotically disabled mouse embryonic fibroblast (MEF) feeder cells. Specifically, trypsinised 700,000 keratinocytes were washed once with sterile PBS and electroporated using the Nucleofector 4D (Lonza, X-module apparatus, kit V4XP-3024, programme DS138, following manufacturer's instructions) with $3 \mu \mathrm{g}$ of the episomal plasmid mix (equimolar mixture of plasmids obtained from Addgene: pCE-hOct3/4, pCEhSK, pCE-hUL, pCE-mp53DD and pCXB-EBNA1). After electroporation, cells were transferred from the cuvette to KGM2 medium (Lonza CC-3103 and CC-4125). Solution was gently mixed and transferred to the six-well plate with feeders. On day 2 (48 hours after electroporation) medium was removed and replaced with fresh KGM2 medium. On the day 4, the medium was switched to standard human embryonic stem cell (hESC) medium (high glucose DMEM with 20\% Knockout Serum Replacement, non-essential amino acids, Glutamax+penstrep (Life Technologies), 2mercaptoethanol $(100 \mu \mathrm{M})$ with $10-20 \mathrm{ng} / \mathrm{mL}$ of FGF2). From day 10 onwards, MEF-CM supplemented with 10-20 $\mathrm{ng} / \mathrm{mL}$ of FGF2 was used. By day 20, iPSC colonies were observed. After day 30, large iPSC colonies were mechanically picked, and expanded using ReLeSR and hESC medium plus ROCK inhibitor (Y-27632, Stem Cell Technologies). The iPSC lines were generated in this way from six unrelated people with DS (QM-DS1, QM-DS2, QM-DS3, QM-DS4, QM-DS5, and QM-DS6, respectively, detailed in Table 1). The iPSCs from a 64-year-old euploid patient with FEOAD caused by DupAPP [21] (QM$\operatorname{Dup} A P P$ ) were generated from peripheral blood mononuclear cells, using the same non-integrational episomal reprogramming vectors as above, and a modified protocol. Specifically, $10^{6}$ PBMCs were electroporated with $3 \mu \mathrm{g}$ of episomal plasmids (equimolar) using program EO-115 and solution p3 on the Amaxa 4D nucleofector. Electroporated PBMCs were transferred into one well of a six-well plate seeded with MEFs in PBMC recovery medium (RPMI supplemented with $200 \mu \mathrm{M}$ 1-thioglycerol (Sigma M6145), $1 \mu \mathrm{M}$ Dexamethasone (Sigma D1756), $2 \mathrm{U} / \mathrm{ml}$ Erythropoietin (R\&D Systems 287-TC-500), $100 \mu \mathrm{g} / \mathrm{ml}$ Holotransferrin (R\&D Systems 2914-HT), $40 \mathrm{ng} / \mathrm{ml}$ IGF-1 (Peprotech 100-11), $10 \mathrm{ng} / \mathrm{ml}$ IL-3 (Peprotech 200-03), $100 \mathrm{ng} / \mathrm{ml} \mathrm{SCF}$ (Peprotech 300-07). After 2 days, $2 \mathrm{ml}$ of
PBMC recovery medium was added to transfected cells. After another 2 days, PBMC recovery medium was replaced every second day with hESC medium supplemented with $10 \mathrm{ng} / \mathrm{ml} \mathrm{FGF2}$. Visible iPSC colonies were mechanically picked and expanded as per the keratinocyte reprogramming protocol. The iPSCs for the isogenic DS model included isogenic clones derived from an individual with DS mosaic for T21 were described by us previously [18]: D21C3, D21C7, D21C9 and T21C5, T21C6, T21C13. These were generated by non-integrationally reprogramming primary skin fibroblasts from a person with mosaic DS, using Sendai virus delivered standard Yamanaka OKSM factors. The total number of iPSC clones generated per patient is listed in Table 1. iPSCs were maintained on Geltrex coated plates and cultured in E8 medium (Life Technologies) supplemented with penicillin/streptomycin. Passaging was carried out using ReLESR and $10 \mu \mathrm{m}$ ROCK inhibitor was included in culture media for 24 hours after passaging.

\section{Cerebral organoids}

Cerebral organoids were generated following a published protocol [19] with the following changes. iPSC lines were first transitioned into feeder free conditions using either mTESR1 or E8 media with geltrex. To form embryoid bodies (EBs), hiPSCs were washed once with PBS, then incubated with Gentle Cell Dissociation Solution (Stemcell Technologies) for 4 mins. This solution was then removed and accutase added and incubated for a further 4 mins. mTESR1/E8 medium at double the volume of accutase was added to the cells and a single cell suspension generated by titurating. Cells were centrifuged to remove accutase and then resuspended in hESC medium supplemented with $4 \mathrm{ng} / \mathrm{ml} \mathrm{FGF2}$ and 50micromolar ROCK inhibitor. In all, 9000 cells were used to form a single EB in each well using a V-shaped ultra low attachment 96 well plate (Corning). Specifically, iPSCs were allowed to form EBs in suspension by culturing for 6 days in hESC medium with low FGF, in non-adherent culture dishes. After 5-7 days, EBs were transferred into a 24 well ultra low attachment plate for neural induction. Neural induction was achieved by culturing for further 5-7 days in DMEM-F12 supplemented with $1 \%$ of each: $\mathrm{N}_{2}$, GlutaMAX, and MEMNEAA, plus $1 \mu \mathrm{g} / \mathrm{ml}$ heparin. Neurally induced EBs showing neuroectodermal "clearing" in brightlight microscopy were embedded in matrigel droplets, and transferred to $6 \mathrm{~cm}$ dishes containing organoid differentiation mediumA, (for 4-5 days), followed by organoid differentiation medium + A [19]. Organoid maturation was carried out with 12-16 organoids per $6 \mathrm{~cm}$ dish on an orbital shaker at $37^{\circ} \mathrm{C}, 5 \% \mathrm{CO}_{2}$. Aliquots of $\mathrm{CM}$ were collected from mature organoids (100-137 days old from day of EB formation), 
3-4 days after feeding (to allow time for cells to secrete products into the culture media). Three completely independent experiments were carried out each time starting from undifferentiated iPSC stage, and CM was collected at 3-4 timepoints in each experiment. CM was immediately frozen and stored at $-80^{\circ} \mathrm{C}$.

For inhibitor treatment, organoids were treated from 20DIV ( 6 days after embedding in matrigel) to 41DIV. $\beta I-$ IV and Compound $\mathrm{E}$ were added freshly to the media before use at final concentrations of $2.5 \mu \mathrm{m}$ and $6 \mathrm{~nm}$, respectively. Media was replaced every 3-4 days during treatment. DMSO of the same volume was used as a vehicle only control.

\section{IP-MS}

CM from organoids was analysed by IP-MS, using a previously described method [20]. The team performing the MS was blinded to the genotypes in all experiments. In exp1, all three independent trisomic clones (T21C6, T21C5, and $\mathrm{T} 21 \mathrm{C} 13$ ) were compared with two independent disomic clones (D21C3 and D21C7), whereas in exp2, two independent trisomic clones (T21C6 and T21C13) were compared with two independent disomic clones (D21C7 and D21C9).

In exp3, a T21C6 clone was compared with the isogenic D21C9 clone, and to hiPSC lines from three unrelated individuals: a DupAPP FEOAD patient (QM-DupAPP), and two unrelated adult people with DS (QM-DS1 and QMDS2).

In all three experiments, IP-MS results for all iPSC lines that were used in a particular experiment are shown. IP-MS results were used to calculate the relative ratios of peptides and these ratios were taken as data points for the statistical comparisons.

IP-MS spectra were also obtained from the CSF samples of people with DS and age-matched normal controls. Peak ratios calculated as described above. The cohorts, methods, and spectra behind these data were previously described [22].

\section{FISH}

FISH on organoid cryosections was performed as described [49]. In brief, slides were rinsed in PBS, rehydrated in 10 mM sodium citrate buffer and incubated in the same buffer at $80^{\circ} \mathrm{C}$ for $20 \mathrm{~min}$. Slides were cooled down and incubated in $2 \times$ saline sodium citrate (SSC) for $5 \mathrm{~min}$ and in $50 \%$ formamide in $2 \times$ SSC for $1 \mathrm{~h}$. After incubation slides were covered with previously prepared hybridization chamber and incubated with $10 \mu \mathrm{L}$ of XA 13/18/21 (D-5607-100-TC, MetaSystems Probes) Probe, protected from the light, at $45^{\circ} \mathrm{C}$ for $2 \mathrm{~h}$, at $80^{\circ} \mathrm{C}$ for $5 \mathrm{~min}$ and for 2 days at $37^{\circ} \mathrm{C}$ in the water bath. Slides were rinsed with $2 \times \mathrm{SSC}$ at $37^{\circ} \mathrm{C}$ $(3 \times 15 \mathrm{~min})$ and with $0.12 \times \mathrm{SSC}$ at $60^{\circ} \mathrm{C}(2 \times 5 \mathrm{~min})$ on the shaker, equilibrated in $2 \times \mathrm{SSC}$ at $37^{\circ} \mathrm{C}$ for $2 \mathrm{~min}$, counterstained with $4^{\prime}, 6$-diamidino-2-phenylindole (DAPI) for $10 \mathrm{~min}$ and covered with Dako Fluorescent Mounting Medium. Fluorescence was captured on Zeiss LSM800 inverted confocal microscope with Airyscan using $\times 63$ oilimmersed objective. Image analysis was performed using IMARIS $\times 64-\mathrm{v} 9.1 .2$. Software (BITPLANE, An Oxford Instruments Co., Zurich, Switzerland). The spots specific for chromosome 21 were labelled in the red spectrum, whereas the spots specific for chromosome 13 were labelled in the green spectrum. More than 500 nuclei from eight different Z-stacks were evaluated for each clone. Based on the observed number of fluorescent hybridization signals, nuclei were assigned to four different categories, namely "one signal", "two signals" "three signals" and ">three signals". Damaged nuclei or overlapped nuclei with other nuclei were not included in scoring.

\section{Immunostaining of organoids}

Cerebral organoids derived from iPSCs and grown for indicated number of DIV were fixed in $4 \%$ paraformaldehyde (PFA), cryoprotected in $30 \%$ sucrose/PBS solution and embedded in OCT. Twenty micron thick sections were cut and mounted on Superfrost Plus slides (Fisher Scientific) for immunostaining.

For immunofluorescent staining, permeabilisation, and blocking was carried out in $3 \%$ donkey serum with $0.2 \%$ TritonX-100 in PBS for $1 \mathrm{~h}$ at room temperature (RT). Primary antibodies (Supplementary Table 3 ) were diluted in $1 \%$ donkey serum with $0.2 \%$ TritonX-100 in PBS and incubated overnight at $4{ }^{\circ} \mathrm{C}$. Following washes with PBS, secondary antibodies (Supplementary Table 4 ) were diluted in $1 \%$ donkey serum with $0.2 \%$ TritonX-100 in PBS and incubated for $2 \mathrm{~h}$ at RT. Following washes with PBS, sections were counterstained with DAPI or DRAQ5 (Supplementary Table 2) for $10 \mathrm{~min}$ at RT, washed again and mounted with DAKO fluorescent mounting medium. As negative controls for all antibodies, secondary antibody only controls were carried out (Supplementary Fig. 15).

\section{Western blot}

For western blots, whole-cell lysates of iPSCs (Fig. 5c, Supplementary Fig. 14a) or organoids (Fig. 7o) were separated in a $10 \%$ acrylamide gel by SDS-PAGE and transferred to a nitrocellulose membrane according to the manufacturers protocols (Bio-Rad). Following a $60 \mathrm{~min}$ incubation in 5\% non-fat milk in TBS-T the membrane was incubated with primary and secondary antibodies (Supplementary Tables 3, 4). Quantification was carried 
out using ImageJ software, strictly using the same membranes re-stained using the antibodies shown. For the protein of interest (BACE2 or TG3), the signal was adjusted to corresponding $\beta$-actin loading control for all samples. Such adjusted values for unedited/untargeted cells $(n=4)$ were set to 1 , and used to calculate the fold change for edited/targeted $(n=4)$ replicates, and the resulting fold change values for pairs run on the same gel were averaged and analysed by student's $t$ test. Membrane stripping between stainings was carried out using Thermo-Fisher stripping solution, following manufacturer's instructions.

\section{AmyloGlo and Thioflavine S staining}

For AmyloGlo staining, OCT embedded slices were rinsed with PBS, and incubated in $70 \%$ ethanol for 5 min at RT, followed by washing with milli Q water for 2 min at RT. Slices were then incubated with AmyloGlo solution for 10 min in the dark at RT, followed by washing in $0.9 \%$ saline solution for $5 \mathrm{~min}$ at RT, and counterstaining with DRAQ5 for $10 \mathrm{~min}$ at RT. Thioflavine $\mathrm{S}$ staining was performed as described [50]. OCT embedded slices were rinsed with PBS and incubated with Thioflavine S solution for $10 \mathrm{~min}$ in the dark at RT, differentiated in $80 \%$ ethanol and counterstained with DRAQ5 for $10 \mathrm{~min}$ at RT, rinsed with PBS and mounted with DAKO fluorescent mounting medium.

\section{Formic acid pre-treatment}

To increase signal of insoluble $\beta$-amyloid material T21C5 4 (96DIV) organoid slices were treated with $87 \%$ Formic acid for $10 \mathrm{~min}$ at RT. After $10 \mathrm{~min}$ formic acid was rinsed three times with PBS and samples were immunostained as described above.

\section{Human brain samples}

PFA-fixed, paraffin-embedded human anonymized postmortem brain samples were obtained from the Brain Bank of the Croatian Institute for Brain Research (CIBR), Institute of Pathology of The Royal London Hospital (IP-RLH) and the South West Dementia Brain Bank (UK) (Supplementary Table 1). Slices were cut at 5-10 $\mu \mathrm{m}$ thickness, and stained using primary antibodies, secondary fluorophorecoupled anti-Ig antibodies and their respective dilutions (Supplementary Tables 3, 4).

\section{Immunohistochemistry and immunofluorescence on human brain tissue}

PFA-fixed, paraffin-embedded 5-10 $\mu$ m thick slides were de-paraffinised by incubation in xylene, rehydrated in a graded series of ethanol and rinsed in PBS for $10 \mathrm{~min}$. For antigen retrieval, the slides were steamed in $0.01 \mathrm{M}$ citrate buffer, pH 6.0 at $100{ }^{\circ} \mathrm{C}$ for $30 \mathrm{~min}$, cooled and rinsed $3 \times$ $10 \mathrm{~min}$ in PBS. On slides used for IHC, endogenous peroxidases were quenched with $0.025 \%$ hydrogen peroxide for $30 \mathrm{~min}$ at RT and rinsed $3 \times 10 \mathrm{~min}$ in PBS. Double immunohistochemical staining was performed "Polink DSMR-Hu A2 Kit for Immunohistochemistry Staining" (GBI Labs, DS202A-18). In brief, Polymer-HRP and AP Double staining kit distinctly labels two different antigens in human tissue, using mouse (GBI-Permanent Red) and rabbit (DAB-brown) antibodies. Single IHC was performed "VECTASTAIN ABC HRP Kit". For immunofluorescence, following antigen retrieval, slides were incubated in blocking/permeabilisation solution $(0.2 \%$ Triton $\mathrm{X}-100$ in PBS $+3 \%$ donkey serum) for $1 \mathrm{~h}$ at RT. The slides were incubated over night at $4{ }^{\circ} \mathrm{C}$ with primary antibodies (Supplementary Table 3 ) in solution $(0.2 \%$ Triton X-100 in PBS $+1 \%$ donkey serum). Next day primary antibodies were rinsed $3 \times 5 \mathrm{~min}$ in PBS and incubated for $2 \mathrm{~h}$ with secondary antibodies (Supplementary Table 4) in $0.2 \%$ Triton X-100 in PBS at RT and rinsed $3 \times 5 \mathrm{~min}$ in PBS. Nuclei were counterstained with DAPI for $10 \mathrm{~min}$, rinsed $3 \times 5 \mathrm{~min}$ in PBS and mounted with Dako Fluorescent Mounting Medium. In order to distinguish the contribution of lipofuscin autofluorescence to the colocalised signals, specificity of primary antibodies (A $\beta \mathrm{x}-34$ and BACE2) has been validated using three different methods: Sudan black B staining (Supplementary Fig. 8a), pre-incubation with BACE2 specific immunogenic peptide (Supplementary Fig. $8 b-e)$ and Lambda $(\lambda)$ scan function on confocal microscope (Supplementary Fig. 8f, g). Three different samples (DS-AD1, DS (28 yrs) pre-AD and euploid sporadic AD (73 yrs) after IHC were stained with $0.1 \%$ Sudan black B in $70 \%$ ethanol for 20 min at RT and analysed on confocal microscope with Airyscan. Sample DS-AD1 was stained with antibodies solution, $12 \mathrm{~h}$ pre-absorbed with BACE2 specific immunogenic peptide, and analysed on confocal microscope and slide scanner. Lambda scan records a series of individual images within a defined wavelength range (in our case from $630 \mathrm{~nm}$ to end of spectrum) and each image was detected at a specific emission wavelength, at $10 \mathrm{~nm}$ intervals. For lambda scan analysis, samples were stained with one primary antibody and labelled with far-red secondary antibody (647). As negative control, we used secondary antibody (647) alone and, as additional negative control, one sample was counterstained with DAPI only, without secondary antibody. As we used a far-red (647) antibody, we analysed expression from 630 $\mathrm{nm}$ to the end of spectrum at $10 \mathrm{~nm}$ intervals. $\mathrm{A} \beta \mathrm{x}-34$ and BACE2 antibodies showed specific peaks, significantly over and above the autofluorescent signal, in all three specific ROI indicated in Fig. 4 and Supplementary Fig. 8 
(intraneuronal fine-vesicles, large intraneuronal spherical granules and extracellular aggregates). DAPI and secondary antibody alone show peaks only at background level. Samples were analysed by: LSM800 Inverted Confocal Microscope with Airyscan (ZEISS), LSM800 Upright Confocal Microscope (ZEISS), LEICA DM6000 CFS, and Axioscan.Z1 Slide Scanner (ZEISS). As negative controls for all antibodies, secondary antibody only controls were carried out (Supplementary Fig. 8h).

\section{Gallyas staining}

For Gallyas staining samples were depariffinised and/or rinsed in PBS, then treated with Ammonium-Silver Nitrate (0.1 $\mathrm{g} \mathrm{NH}_{4} \mathrm{NO}_{3}, 0.1 \mathrm{~g} \mathrm{AgNO}_{3}, 0.3 \mathrm{~mL} 4 \% \mathrm{NaOH}$ ) solution for $30 \mathrm{~min}$ protected from the light, rinsed with $0.5 \%$ acetic acid $(3 \times 3 \mathrm{~min})$ and placed in developer solution for 5-30 min. Developer solution was made from three stock solutions: $25 \mathrm{ml}$ of Solution A $\left(50 \mathrm{~g} \mathrm{Na} \mathrm{CO}_{3}+1000 \mathrm{~mL}\right.$ distilled water), $7.5 \mathrm{ml}$ of Solution $\mathrm{B}\left(2 \mathrm{~g} \mathrm{NH} \mathrm{NHO}_{3}+2 \mathrm{~g}\right.$ $\mathrm{AgNO}_{3}+10 \mathrm{~g}$ Tungstosalicic acid hydrate $+1000 \mathrm{~mL}$ distilled water) and $17.5 \mathrm{ml}$ of Solution $\mathrm{C}\left(2 \mathrm{~g} \mathrm{NH}_{4} \mathrm{NO}_{3}+2\right.$ $\mathrm{g} \mathrm{AgNO}_{3}+10 \mathrm{~g}$ Tungstosalicic acid hydrate $+7.3 \mathrm{ml} \mathrm{37 \%}$ formaldehyde solution $+1000 \mathrm{~mL}$ distilled water). After developer solution samples were rinsed in water and placed in destaining solution $\left(30 \mathrm{~g} \mathrm{~K}_{2} \mathrm{CO}_{3}+55 \mathrm{~g}\right.$ EDTA-Na $2+25$ $\mathrm{g} \mathrm{FeCl}_{3}+120 \mathrm{~g} \mathrm{Na}_{2} \mathrm{~S}_{2} \mathrm{O}_{3}+20 \mathrm{~g} \mathrm{KBr}+1000 \mathrm{~mL}$ distilled water). Finally, samples were rinsed two times in $0.5 \%$ acetic acid. After staining samples were rinsed in water, dehydrated in a graded series of ethanol, cleared in HistoClear and mounted with Histomount mounting medium. Samples were scanned by NanoZoomer 2.0RS (HAMAMATSU).

\section{Image analysis}

Immunofluorescent stains of $20 \mu \mathrm{m}$ thick slices are shown as maximal projections captured on Zeiss LSM800 upright confocal microscope using $\times 63$ oil-immersed objective. Image analysis was performed using IMARIS x64-v9.3.1. Software (BITPLANE, An Oxford Instruments Co., Zurich, Switzerland). Quantification was performed blinded to the genotype, on five independent images representing three individual organoids per genotype, and containing 3000-4000 cells per image. Only images within the "cortical" part of the organoid were considered for analysis. For quantification of protein/peptide markers, total fluorescence intensity of positive signals for each wavelength for a given antibody was normalised to the total fluorescence intensity for MAP2 as a pan-neuronal marker.

For colocalisation calculations: image analysis was performed using IMARIS software. Pairwise Pearson's coefficient of colocalised volume for a pair of co-stained antibodies with contrasting fluorescence wavelengths was automatically calculated by the IMARIS software on 3-8 images from three independent organoids, per any given antibody combination, using a maximal projection through the entire $\mathrm{z}$-stack.

\section{A new FRET-assay for the detection of A $\beta D P$ activity generating $A \beta 1-34$}

We designed and synthesized a new FRET-based peptide containing the fluorophore at one end and the quencher at the other end, and spanning the A $\beta 34$ site in its middle. The exact peptide design is under discussion for intellectual property protection. The FRET (BACE2 R\&D Systems, not based on amyloid sequence) control peptide $(10 \mu \mathrm{M})$ (not shown) or the newly designed FRET A $\beta 34$ site peptide (10 $\mu \mathrm{M}$ ) were digested at $37^{\circ} \mathrm{C}$ by human BACE2 (R\&D systems, $1 \mathrm{ng} / \mu \mathrm{L})$ in presence or absence of the stated inhibitors for $2 \mathrm{~h}$. Three replicates per inhibitor per concentration were used. Enzyme activity was defined by measuring the fluorescence increase before and after the incubation. After blank-subtracted fluorescence units were normalized to the control digest, one-way analysis of variance (ANOVA) was performed. $P$ values were calculated with a post hoc Bonferroni correction for multiple comparisons (only pairs relative to the untreated control were simultaneously compared). Error bars represent standard error.

\section{Statistical analysis}

Initial analysis was carried out using Microsoft Excel to calculate two-tailed student $t$ tests. Additional Holm-Bonferroni correction was carried out using the Excel macro from ref. [51]. For all multiple comparison analysis, ANOVA and Holm-Bonferroni calculations were performed at http://astatsa.com/OneWay_Anova_with_ TukeyHSD/, or using GraphPad Prism 8.4.1 software.

\section{SNP arrays}

iPSC lines genome integrity: genomic DNA was isolated from iPSCs using standard column kits. DNA of all iPSC lines shown in the manuscript were re-analysed at the similar passage used for the derivation of organoids using SNP arrays Illumina OmniExpress v1.1 chips and analysis performed in Genome Studio 2.0 software. Following CRISPR editing, T21C5 47 was assessed by SNP array and no genomic alternations were detected compared with the parental C5 iPSC clone. See the raw data from this for chromosome 21 array, the rest are not shown for the lack of space, data available on request). The genome integrity of the isogenic iPSC clones was previously published [18] (but was repeated here as described above). No additional 
rearrangements owing to re-programming or passaging were observed.

The cohort of people with DS has been described in recent reports $[52,53]$. In brief, participants donated DNA samples and had detailed cognitive and clinical assessments to determine dementia status [54]. Age of dementia diagnosis was established and shown in Table 1. Genotyping was done in the UCL Genomics Centre using Human OmniExpressExome v1.2,v1.3,v1.4 beadchips. SNP clustering and genotyping, was undertaken using GenomeStudio (Illumina, San Diego, CA, USA). Manual reclustering for Chr 21 SNPs was done using GenomeStudio module v1.9.4 polyploidy-genotyping (http://res.illumina.com/ documents/products/technotes/technote_genomestudio_ polyploid_genotyping.pdf).

\section{Quantitative paralogous amplification- pyrosequencing}

Quantitative paralogous amplification-pyrosequencing was carried out based on the published method [55]. This method takes advantage of the existence of identical sequences on chromosome 21 and one other autosome, allowing amplification of both loci with a single primer pair. Paralogous sequence mismatches in amplified products from chromosome 21 (GABPA and ITSN) can be quantified relative to their paralogous regions on chromosome 7 and 5 , respectively. As such, trisomic cells show a 60:40 ratio for the paralogous sequence, whereas disomic cells produce a 50:50 ratio. Primers used for amplification and pyrosequencing are listed in Supplementary Table 5. Pyrosequencing was performed on the Pyromark Q48 machine (Qiagen) following standard procedures.

\section{CRISPR/SpCas9-HF1 editing of the BACE2 locus}

The guide-RNA (gRNA) targeting BACE2 Exon 3 was cloned into a vector containing the high fidelity SpCas9HF1 [56] and blasticidin S resistance gene. The complete plasmid was delivered via Lipofectamine3000 to a trisomic iPSC clone T21C5 (full official name NIZEDSM1iT21-C5), which was described and characterized in a previous report [18]. Untransfected iPSCs were removed by treatment with blasticidin $(2 \mu \mathrm{g} / \mathrm{ml}$ for $48 \mathrm{~h})$. Individual colonies were picked and further sub cloned by limiting dilution to achieve clonal cell lines. DNA was purified from individual clones, PCR amplified and sequenced by Sanger Sequencing. Sequences were analysed in Mutation Surveyor (V3.1.0) and "Tracking InDels by dEcomposition (TIDE)" (TIDE V 2.0.1, Desktop Genetics). TIDE analysis of the CRISPR-targeted clone 2.3.5 DNA sequence gave a score of $65 \%$ of the wt read remaining (not shown). The quality of the gRNA was assessed using two different prediction software platforms: CCTop online software [57], and the MIT online platform (http://crispr.mit.edu/). The same two software platforms were used to predict the off-target sites. Neither platform found any off-targets with 0 , 1 , or 2 mismatches. The top 10 CCTop-predicted sites were PCR amplified in both $\Delta 7$ and WT clones, then sequenced by Sanger Sequencing to rule out off-target events. No differences in the sequence were found.

\section{shRNA targeting of BACE2 in iPSCs}

One day after plating, small colonies of feeder free iPSCs were transduced with lentiviral shRNA particles targeting BACE2 (sc-29776) or a non-targeting control (sc-108080). Lentiviral particles were obtained from Santa Cruz Biotechnology Inc. The lentiviral particles for BACE2 contain three different shRNAs targeting human BACE2. In total, 5000 infectious units (IFU) of virus were added per 12-well, and incubated overnight. The following day, viral media was removed and replaced with fresh media. Four days after transduction, stably transduced cells were selected by treatment with $0.5 \mu \mathrm{g} / \mathrm{ml}$ puromycin for 14 days, passaging as required. Following this, iPSCs were maintained in 0.3 $\mu \mathrm{g} / \mathrm{ml}$ puromycin. Knockdown of BACE2 was confirmed by western blot (Supplementary Fig. 14a).

\section{Protein isolation from cortical organoids}

Organoids were collected at specified durations in culture (expressed as DIV) and washed twice with ice-cold PBS. The samples were resuspended in ice-cold NP-40 Buffer (150 mm NaCl, 1\% NP-40, $50 \mathrm{~mm}$ Tris pH8) containing EDTA free protease inhibitors (complete cocktail, Roche) and lysed using a $1 \mathrm{ml}$ tissue homogenizer (Fisher). Each sample was centrifuged at $10,000 \mathrm{rpm}$ for 10 minutes at $4{ }^{\circ} \mathrm{C}$ and the homogenates were stored at $-80^{\circ} \mathrm{C}$. Protein concentration was determined using the bicinchoninic acid method (Pierce).

\section{Detection of fibrillary material from organoids by transmission electron microscopy (TEM)}

Organoids were lysed following the same procedure for protein extraction, however, samples were initially spun at $20,000 \times g$ for 20 minutes at $4{ }^{\circ} \mathrm{C}$. Following the first centrifugation, supernatants were removed and kept on ice. The remaining cell pellets were resuspended in $5 \times$ weight/ volume buffer (10 mm Tris-HCL pH7.5, 0.8 $\mathrm{M} \mathrm{NaCl}$, and $10 \%$ sucrose) [58] containing proteases inhibitor and spun at $20,000 \times g$ for 20 minutes at $4{ }^{\circ} \mathrm{C}$. An equal volume of supernatant 1 was added to the supernatant from the second centrifugation step. $1 \% \mathrm{~N}$-lauroysarcosinate (weight/ volume) was added and the samples were rocked at RT for 
1 hour. The samples were ultra-centrifuged at $100,000 \times g$ for 1 hour at $4{ }^{\circ} \mathrm{C}$. The supernatant was decanted and the sarkosyl-insoluble pellet was resuspended in ice-cold PBS prior to imaging. The samples were deposited on to glowdischarged 400 mesh formvar/carbon film-coated copper grids, negatively stained with a $2 \%$ aqueous (w/v) uranyl acetate solution and then immediately analysed at $100 \mathrm{kV}$ using a JEOL TEM1010 equipped with a Gatan Orius camera.

\section{TEM analysis of synthetic $A \beta 1-40$ fibrils in vitro}

Synthetic A $\beta$ peptide powder (China peptides) was treated with 1,1,1,3,3,3-hexafluoro-2-propanol and lyophilized. The peptide was then dissolved in $20 \mu \mathrm{L}$ of $100 \mathrm{~mm} \mathrm{NaOH}$ and then diluted with buffer. A $50 \mu \mathrm{M}$ stock of this monomeric $\mathrm{A} \beta$ peptide was grown at $37^{\circ} \mathrm{C}$ shaking at $180 \mathrm{rpm}$ for 48-60 hours before recording the TEM images. In all, 4 $\mu \mathrm{L}$ of extract was added to a $15 \mathrm{~nm}$ thick, lacey carbon on 300 mesh grid (glow-discharged) for 2 minutes followed by negative staining with $2 \%$ uranyl acetate for 1 minute and then air dried. The grids were then viewed under FEI T12, $120 \mathrm{kV}$ Transmission electron microscope equipped with a $4 \mathrm{~K} \mathrm{CCD}$ camera (FEI) at $\times 30,000$ magnification under lowdose conditions.

\section{Data availability}

All data that support the findings described in this study are available within the manuscript and the related supplementary information, and from the corresponding authors upon reasonable request.

Acknowledgements DN's work was funded by the Singapore National Medical Research Council (NMRC/CIRG/1438/2015), Singapore Ministry of Education Academic Research Fund Tier 2 grants (2015T2-1-023 \& 2015-T2-2-119), The Wellcome Trust Collaborative Award in Science 217199/Z/19/Z, and by the "Research Cooperability" Programme of the Croatian Science Foundation PZS-2019-02-4277 and EU-JPND-"Heroes" and "CoEN" Consortia. DN, JH, and AS all received funding as part of The Wellcome Trust "LonDownS Consortium" Strategic Funding Award (098330/Z/12/Z) (UK), and JH received funding from the Dementia Research Institute, an anonymous foundation and the Dolby foundation. HZ is a Wallenberg Academy Fellow supported by grants from the Swedish Research Council, the European Research Council, Swedish State Support for Clinical Research (ALFGBG-720931) the UK Dementia Research Institute at UCL. KB holds the Torsten Söderberg Professorship in Medicine at the Royal Swedish Academy of Sciences, and is supported by the Swedish Research Council (\#2017-00915), the Swedish Alzheimer Foundation (\#AF-742881), Hjärnfonden, Sweden (\#FO2017-0243), and the Swedish State Support for Clinical Research (\#ALFGBG-715986). AM was awarded a William Harvey Academy Fellowship, co-funded by the People Programme (Marie Curie Actions) of the European Union's Seventh Framework Programme (FP7/2007-2013) under REA grant agreement no. 608765. JNF received a fellowship from the Singapore National Research Foundation (NRF-NRFF2016-03). ARL received funding from the Fondation pour la Recherche Médicale (FRM). JGh and AR are supported by the BrightFocus Foundation (A2015275S), and NIH grant AG059695. The work of ŽK, Gك̌, IK, and DM research was co-financed by the Scientific Centre of Excellence for Basic, Clinical and Translational Neuroscience (project "Experimental and clinical research of hypoxic-ischemic damage in perinatal and adult brain"; GA KK01.1.1.01.0007 funded by the European Union through the European Regional Development Fund). ŽK is also supported by the Adris Foundation. GŠ is also supported by the Croatian Science Foundation (HRZZ IP-2019-04-3584). DM is supported by the Croatian Science Foundation (IP-2016-06-9451). The South West Dementia Brain Bank is jointly funded by Alzheimer's Research UK and Alzheimer's Society, and is supported by BRACE (Bristol Research into Alzheimer's and Care of the Elderly) and the Medical Research Council, UK. All unique materials will be made available for academic and non-commercial research purposes. We acknowledge Balakrishnan Kannan in the LKC imaging facility for his assistance. The authors thank Géraldine Joly-Hélas, Pascal Chambon, Željka Punčec, Ana Bosak, and Danica Budinščak for technical help, Marie Loh and Jacqueline Tai for the use of pyrosequencer, Moses Tandiono for help with SNP array experiments, Selina Wray for some antibodies, and Anna Barron and Madeline Lancaster for advice. We are grateful to Maria Grazia Spillantini for advice, critical comments, and AT100 antibody.

LonDownS Consortium Andre Strydom ${ }^{19,20}$, Elizabeth Fisher ${ }^{21}$, Frances Wiseman ${ }^{21}$, Dean Nizetic ${ }^{22,23}$, John Hardy ${ }^{24,25}$, Victor Tybulewicz $^{26,27}$, Annette Karmiloff-Smith ${ }^{28}$

${ }^{19}$ Department of Forensic and Neurodevelopmental Sciences, Institute of Psychiatry, Psychology and Neuroscience, King's College London, London, UK; ${ }^{20}$ Division of Psychiatry, University College London, London, UK; ${ }^{21}$ Department of Neurodegenerative Disease, UCL Institute of Neurology, London, UK; ${ }^{22}$ Blizard Institute, Barts and the London School of Medicine, Queen Mary University of London, London, UK; ${ }^{23}$ Lee Kong Chian School of Medicine, Nanyang Technological University, Singapore, Singapore; ${ }^{24}$ Reta Lila Weston Institute, Institute of Neurology, University College London, London, London, UK; ${ }^{25} \mathrm{UK}$ Dementia Research Institute, University College London, London, UK; ${ }^{27}$ Francis Crick Institute, London, UK; ${ }^{26}$ Department of Medicine, Imperial College, London, UK; ${ }^{28}$ Birkbeck University, London, UK

Author contributions IA, PAG, AM, EP, JH, HZ, and DN contributed to the concept and design, the acquisition, analysis and interpretation, and drafting of manuscript. AS, DK, JGr, SHav, NRD, and KB contributed to the concept and design and to acquisition and analysis and interpretation. YJY, GB, RH, CS, SHam, DW, ARL, HK, JEM, AR, JGh, ŽK, GŠ , and LC contributed to acquisition of data. GG, KYM, $\mathrm{RB}, \mathrm{NLO}, \mathrm{MP}, \mathrm{KP}, \mathrm{MT}$, DLB, and DM contributed to acquisition, analysis, and interpretation of data. EG, XS, HS, and JNF contributed to acquisition and analysis of data. EV contributed to analysis and interpretation. IK and PTF contributed to the concept and design and the acquisition of data.

\section{Compliance with ethical standards}

Conflict of interest HZ has served at scientific advisory boards for CogRx, Roche Diagnostics, Samumed, and Wave and is a co-founder of Brain Biomarker Solutions in Gothenburg AB, a GU Venturesbased platform company at the University of Gothenburg (all unrelated to the submitted work). JEM chairs the MNDA Research Advisory Board and is a director of Biomoti, a cancer drug-delivery company. 
Publisher's note Springer Nature remains neutral with regard to jurisdictional claims in published maps and institutional affiliations.

Open Access This article is licensed under a Creative Commons Attribution 4.0 International License, which permits use, sharing, adaptation, distribution and reproduction in any medium or format, as long as you give appropriate credit to the original author(s) and the source, provide a link to the Creative Commons license, and indicate if changes were made. The images or other third party material in this article are included in the article's Creative Commons license, unless indicated otherwise in a credit line to the material. If material is not included in the article's Creative Commons license and your intended use is not permitted by statutory regulation or exceeds the permitted use, you will need to obtain permission directly from the copyright holder. To view a copy of this license, visit http://creativecommons. org/licenses/by/4.0/.

\section{References}

1. De Strooper B, Vassar R, Golde T. The secretases: enzymes with therapeutic potential in Alzheimer disease. Nat Rev Neurol. 2010;6:99-107.

2. Hardy J, Selkoe DJ. The amyloid hypothesis of Alzheimer's disease: progress and problems on the road to therapeutics. Science. 2002;297:353-6.

3. Masters CL, Simms G, Weinman NA, Multhaup G, McDonald BL, Beyreuther K. Amyloid plaque core protein in Alzheimer disease and Down syndrome. Proc Natl Acad Sci USA. 1985;4245-9.

4. Mawuenyega KG, Sigurdson W, Ovod V, Munsell L, Kasten T, Morris JC, et al. Decreased clearance of CNS beta-amyloid in Alzheimer's disease. Science. 2010;330:1774.

5. Scheltens P, Blennow K, Breteler MM, de Strooper B, Frisoni GB, Salloway S, et al. Alzheimer's disease. Lancet. 2016;388:505-17.

6. Hithersay R, Startin CM, Hamburg S, Mok KY, Hardy J, Fisher EMC, et al. Association of Dementia With Mortality Among Adults With Down Syndrome Older Than 35 Years. JAMA Neurol. 2018;76:52-160.

7. Wiseman FK, Al-Janabi T, Hardy J, Karmiloff-Smith A, Nizetic $\mathrm{D}$, Tybulewicz VL, et al. A genetic cause of Alzheimer disease: mechanistic insights from Down syndrome. Nat Rev Neurosci. 2015;16:564-74.

8. Willem M, Tahirovic S, Busche MA, Ovsepian SV, Chafai M, Kootar S, et al. eta-Secretase processing of APP inhibits neuronal activity in the hippocampus. Nature. 2015;526:443-7.

9. Vassar R, Bennett BD, Babu-Khan S, Kahn S, Mendiaz EA, Denis $\mathrm{P}$, et al. Beta-secretase cleavage of Alzheimer's amyloid precursor protein by the transmembrane aspartic protease BACE. Science. 1999;286:735-41.

10. Vassar R, Kuhn PH, Haass C, Kennedy ME, Rajendran L, Wong PC, et al. Function, therapeutic potential and cell biology of BACE proteases: current status and future prospects. J Neurochem. 2014;130:4-28.

11. Yan R. Physiological Functions of the beta-Site Amyloid Precursor Protein Cleaving Enzyme 1 and 2. Front Mol Neurosci 2017;10:97 (2017).

12. Abdul-Hay SO, Sahara T, McBride M, Kang D, Leissring MA. Identification of BACE2 as an avid ss-amyloid-degrading protease. Mol Neurodegener. 2012;7:46.

13. Fluhrer R, Capell A, Westmeyer G, Willem M, Hartung B, Condron MM, et al. A non-amyloidogenic function of BACE-2 in the secretory pathway. J Neurochem. 2002;81:1011-20.

14. Shi XP, Tugusheva K, Bruce JE, Lucka A, Wu GX, Chen-Dodson $\mathrm{E}$, et al. Beta-secretase cleavage at amino acid residue 34 in the amyloid beta peptide is dependent upon gamma-secretase activity. J Biol Chem. 2003;278:21286-94.
15. Sun X, He G, Song W. BACE2, as a novel APP theta-secretase, is not responsible for the pathogenesis of Alzheimer's disease in Down syndrome. FASEB J. 2006;20:1369-76.

16. Salehi A, Delcroix JD, Belichenko PV, Zhan K, Wu C, Valletta JS, et al. Increased App expression in a mouse model of Down's syndrome disrupts NGF transport and causes cholinergic neuron degeneration. Neuron. 2006;51:29-42.

17. Teller JK, Russo C, DeBusk LM, Angelini G, Zaccheo D, DagnaBricarelli F, et al. Presence of soluble amyloid beta-peptide precedes amyloid plaque formation in Down's syndrome. Nature medicine. 1996;2:93-5.

18. Murray A, Letourneau A, Canzonetta C, Stathaki E, Gimelli S, Sloan-Bena F, et al. Isogenic Induced Pluripotent Stem Cell Lines from an Adult with Mosaic Down Syndrome Model Accelerated neuronal Ageing and Neurodegeneration. Stem Cell. 2015;33:77-84.

19. Lancaster MA, Knoblich JA. Generation of cerebral organoids from human pluripotent stem cells. Nature protocols. 2014;9:2329-40.

20. Portelius E, Tran AJ, Andreasson U, Persson R, Brinkmalm G, Zetterberg $\mathrm{H}$, et al. Characterization of amyloid beta peptides in cerebrospinal fluid by an automated immunoprecipitation procedure followed by mass spectrometry. J Proteome Res. 2007;6:4433-9.

21. Rovelet-Lecrux A, Hannequin D, Raux G, Le Meur N, Laquerriere A, Vital A, et al. APP locus duplication causes autosomal dominant early-onset Alzheimer disease with cerebral amyloid angiopathy. Nature genetics. 2006;38:24-6.

22. Portelius E, Holtta M, Soininen H, Bjerke M, Zetterberg H, Westerlund A, et al. Altered cerebrospinal fluid levels of amyloid beta and amyloid precursor-like protein 1 peptides in Down's syndrome. Neuromolecular Med. 2014;16:510-6.

23. Cabrera E, Mathews P, Mezhericher E, Beach TG, Deng J, Neubert TA, et al. Abeta truncated species: Implications for brain clearance mechanisms and amyloid plaque deposition. Biochim Biophys Acta. 2018;1864:208-25.

24. Yu WH, Cuervo AM, Kumar A, Peterhoff CM, Schmidt SD, Lee $\mathrm{JH}$, et al. Macroautophagy-a novel Beta-amyloid peptide-generating pathway activated in Alzheimer's disease. J Cell Biol. 2005;171:87-98.

25. Schmued L, Raymick J, Tolleson W, Sarkar S, Zhang YH, BellCohn A. Introducing Amylo-Glo, a novel fluorescent amyloid specific histochemical tracer especially suited for multiple labeling and large scale quantification studies. J Neurosci Methods. 2012;209:120-6.

26. Mok KY, Jones EL, Hanney M, Harold D, Sims R, Williams J, et al. Polymorphisms in BACE2 may affect the age of onset Alzheimer's dementia in Down syndrome. Neurobiol Aging. 2014;35:1513 e1511-1515.

27. Ahmed RR, Holler CJ, Webb RL, Li F, Beckett TL, Murphy MP. BACE1 and BACE2 enzymatic activities in Alzheimer's disease. J Neurochem. 2010;112:1045-53.

28. Bennett BD, Babu-Khan S, Loeloff R, Louis JC, Curran E, Citron $\mathrm{M}$, et al. Expression analysis of BACE2 in brain and peripheral tissues. J Biol Chem. 2000;275:20647-51.

29. Holler CJ, Webb RL, Laux AL, Beckett TL, Niedowicz DM, Ahmed RR, et al. BACE2 expression increases in human neurodegenerative disease. Am J Pathol. 2012;180:337-50.

30. Stockley JH, Ravid R, O'Neill C. Altered beta-secretase enzyme kinetics and levels of both BACE1 and BACE2 in the Alzheimer's disease brain. FEBS Lett. 2006;580:6550-60.

31. Dobrowolska Zakaria JA, Vassar RJ. A promising, novel, and unique BACE1 inhibitor emerges in the quest to prevent Alzheimer's disease. EMBO Mol Med. 2018;10:e9717.

32. Neumann U, Ufer M, Jacobson LH, Rouzade-Dominguez ML, Huledal G, Kolly C, et al. The BACE-1 inhibitor CNP520 for prevention trials in Alzheimer's disease. EMBO Mol Med. 2018;10:pii:e9316. 
33. Vassar R. BACE1 inhibitor drugs in clinical trials for Alzheimer's disease. Alzheimers Res Ther. 2014;6:89.

34. Dominguez D, Tournoy J, Hartmann D, Huth T, Cryns K, Deforce $\mathrm{S}$, et al. Phenotypic and biochemical analyses of BACE1- and BACE2-deficient mice. J Biol Chem. 2005;280:30797-806.

35. Myllykangas L, Wavrant-De Vrieze F, Polvikoski T, Notkola IL, Sulkava R, Niinisto L, et al. Chromosome 21 BACE2 haplotype associates with Alzheimer's disease: a two-stage study. J Neurol Sci. 2005;236:17-24.

36. Rovelet-Lecrux A, Charbonnier C, Wallon D, Nicolas G, Seaman $\mathrm{MN}$, Pottier $\mathrm{C}$, et al. De novo deleterious genetic variations target a biological network centered on Abeta peptide in early-onset Alzheimer disease. Mol Psychiatry. 2015;20:1046-56.

37. Evin G. Future Therapeutics in Alzheimer's Disease: Development Status of BACE Inhibitors. BioDrugs. 2016;30:173-94.

38. Hernandez-Guillamon M, Mawhirt S, Blais S, Montaner J, Neubert TA, Rostagno A, et al. Sequential Amyloid-beta Degradation by the Matrix Metalloproteases MMP-2 and MMP-9. J Biol Chem. 2015;290:15078-91.

39. Saido T, Leissring MA. Proteolytic degradation of amyloid betaprotein. Cold Spring Harb Perspect Med. 2012;2:a006379.

40. Lee MH, Siddoway B, Kaeser GE, Segota I, Rivera R, Romanow WJ, et al. Somatic APP gene recombination in Alzheimer's disease and normal neurons. Nature. 2018;563:39-45.

41. Wiseman FK, Pulford LJ, Barkus C, Liao F, Portelius E, Webb R, et al. Trisomy of human chromosome 21 enhances amyloid-beta deposition independently of an extra copy of APP. Brain. 2018.

42. Azkona G, Levannon D, Groner Y, Dierssen M. In vivo effects of APP are not exacerbated by BACE2 co-overexpression: behavioural characterization of a double transgenic mouse model. Amino Acids. 2010;39:1571-80.

43. Gonzalez C, Armijo E, Bravo-Alegria J, Becerra-Calixto A, Mays CE, Soto C. Modeling amyloid beta and tau pathology in human cerebral organoids. Mol Psychiatry. 2018;23:2363-74.

44. Mann DMA, Davidson YS, Robinson AC, Allen N, Hashimoto T, Richardson A, et al. Patterns and severity of vascular amyloid in Alzheimer's disease associated with duplications and missense mutations in APP gene, Down syndrome and sporadic Alzheimer's disease. Acta Neuropathol. 2018;136:569-87.

45. Ovchinnikov DA, Korn O, Virshup I, Wells CA, Wolvetang EJ. The Impact of APP on Alzheimer-like Pathogenesis and Gene
Expression in Down Syndrome iPSC-Derived Neurons. Stem Cell Reports. 2018;11:32-42.

46. Cuervo AM, Dice JF. Unique properties of lamp2a compared to other lamp2 isoforms. J Cell Sci. 2000;113:4441-50.

47. Kaushik S, Cuervo AM. The coming of age of chaperone-mediated autophagy. Nat Rev Mol Cell Biol. 2018;19:365-81.

48. Park JS, Kim DH, Yoon SY. Regulation of amyloid precursor protein processing by its KFERQ motif. BMB Rep. 2016;49:337-42.

49. Solovei I, Cremer M. 3D-FISH on cultured cells combined with immunostaining. Methods Mol Biol. 2010;659:117-26.

50. Espuny-Camacho I, Arranz AM, Fiers M, Snellinx A, Ando K, Munck S, et al. Hallmarks of Alzheimer's Disease in Stem-CellDerived Human Neurons Transplanted into Mouse Brain. Neuron. 2017;93:1066-81 e1068.

51. Gaetano J. Holm-Bonferroni sequential correction: An EXCEL calculator (1.2) [Microsoft Excel workbook]. 2013; https://doi. org/10.13140/RG.2.1.3920.0481.

52. Startin CM, Hamburg S, Hithersay R, Al-Janabi T, Mok KY, Hardy J, et al. Cognitive markers of preclinical and prodromal Alzheimer's disease in Down syndrome. Alzheimers Dement. 2019;15:245-57.

53. Strydom A, Heslegrave A, Startin CM, Mok KY, Hardy J, Groet $\mathrm{J}$, et al. Neurofilament light as a blood biomarker for neurodegeneration in Down syndrome. Alzheimers Res Ther. 2018;10:39.

54. Startin CM, Hamburg S, Hithersay R, Davies A, Rodger E, Aggarwal N, et al. The LonDownS adult cognitive assessment to study cognitive abilities and decline in Down syndrome. Wellcome Open Res. 2016;1:11.

55. Deutsch S, Choudhury U, Merla G, Howald C, Sylvan A, Antonarakis SE. Detection of aneuploidies by paralogous sequence quantification. J Med Genet. 2004;41:908-15.

56. Kleinstiver BP, Pattanayak V, Prew MS, Tsai SQ, Nguyen NT, Zheng Z, et al. High-fidelity CRISPR-Cas9 nucleases with no detectable genome-wide off-target effects. Nature. 2016;529:490-5.

57. Stemmer M, Thumberger T, Del Sol Keyer M, Wittbrodt J, Mateo JL. CCTop: An Intuitive, Flexible and Reliable CRISPR/Cas9 Target Prediction Tool. PloS ONE. 2015;10:e0124633.

58. Goedert M, Spillantini MG, Cairns NJ, Crowther RA. Tau proteins of Alzheimer paired helical filaments: abnormal phosphorylation of all six brain isoforms. Neuron. 1992;8:159-68.

\section{Affiliations}

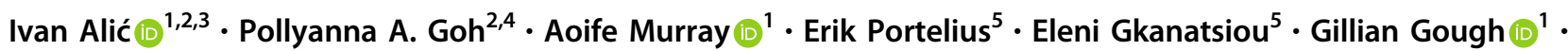
Kin Y. Mok $\mathbb{1}^{4,6} \cdot$ David Koschut $^{1} \cdot$ Reinhard Brunmeir ${ }^{1} \cdot$ Yee Jie Yeap ${ }^{1} \cdot$ Niamh L. O'Brien $^{2,4} \cdot$ Jürgen Groet $^{2,4}$. Xiaowei Shao $\mathbb{D}^{1} \cdot$ Steven Havlicek ${ }^{7}$ - N. Ray Dunn ${ }^{1,8} \cdot$ Hlin Kvartsberg $^{5} \cdot$ Gunnar Brinkmalm $\oplus^{5}$. Rosalyn Hithersay ${ }^{4,9,10}$. Carla Startin $\mathbb{1}^{4,9} \cdot$ Sarah Hamburg ${ }^{4,9}$. Margaret Phillips ${ }^{11}$ - Konstantin Pervushin ${ }^{11}$. Mark Turmaine $^{12}$ - David Wallon ${ }^{13} \cdot$ Anne Rovelet-Lecrux $^{13} \cdot$ Hilkka Soininen ${ }^{14}{ }^{14}$. Emanuela Volpi (i) $^{15}$. Joanne E. Martin $\left(^{2}\right)^{2} \cdot$ Jia Nee Foo ${ }^{1,7} \cdot$ David L. Becker ${ }^{1} \cdot$ Agueda Rostagno $^{16} \cdot$ Jorge Ghiso $^{16} \cdot$ Željka Krsnik $^{17} \cdot$ Goran Šimić $^{17}$ - Ivica Kostović ${ }^{17}$ - Dinko Mitrečić $\mathbb{D}^{17} \cdot$ LonDownS Consortium $^{4}$ • Paul T. Francis ${ }^{18}$. Kaj Blennow $\mathbb{D}^{5} \cdot$ Andre Strydom $\mathbb{1}^{4,9,10} \cdot$ John Hardy ${ }^{4,6} \cdot$ Henrik Zetterberg ${ }^{5,6} \cdot$ Dean Nižetić $\mathbb{1}^{1,2,4}$

1 Lee Kong Chian School of Medicine, Nanyang Technological University, Singapore 308232, Singapore

2 The Blizard Institute, Barts \& The London School of Medicine, Queen Mary University of London, London E1 2AT, UK
3 Department of Anatomy, Histology and Embryology, Faculty of Veterinary Medicine, University of Zagreb, 10000 Zagreb, Croatia

4 LonDownS Consortium, London, UK 
5 Department of Psychiatry and Neurochemistry, Institute of Neuroscience and Physiology, The Sahlgrenska Academy at the University of Gothenburg, Gothenburg S-405 30, Sweden

6 Dementia Research Institute \& Reta Lila Weston Institute, Institute of Neurology, University College London, London WC1N 3BG, UK

7 Genome Institute of Singapore, Agency for Science, Technology and Research (A*STAR), Singapore 138672, Singapore

8 Institute of Medical Biology, Agency for Science, Technology and Research (A*STAR), Singapore 138648, Singapore

9 Division of Psychiatry, University College London, London WC1E 6BT, UK

10 Department of Forensic and Neurodevelopmental Sciences, Institute of Psychiatry, Psychology \& Neuroscience, King's College London, London SE5 8AF, UK

11 School of Biological Sciences, Nanyang Technological University, Singapore 639798, Singapore
12 Division of Biosciences, University College London, Gower Street, London WC1E 6BT, UK

13 Normandie Univ, UNIROUEN, Inserm U1245 and Rouen University Hospital, Department of Neurology and CNR-MAJ, F 76000, Normandy Center for Genomic and Personalized Medicine, Rouen, France

14 University of Eastern Finland, Institute of Clinical Medicine/ Neurology, Kuopio FI-70211, Finland

15 School of Life Sciences, University of Westminster, London W1W 6UW, UK

16 Department of Pathology \& Department of Psychiatry, New York University School of Medicine, New York, NY 10016, USA

17 Croatian Institute for Brain Research, School of Medicine, University of Zagreb, 10000 Zagreb, Croatia

18 Wolfson Centre for Age-Related Diseases, King's College London, London SE1 1UL, UK 